\title{
Stable elastic knots with no self-contact
}

\author{
Derek E. Moulton \\ Mathematical Institute, University of Oxford, U.K. \\ Paul Grandgeorge \\ Sorbonne Université, UPMC Univ Paris 06, CNRS, UMR 7190 \\ Institut Jean Le Rond d'Alembert,F-75005 Paris, France \\ Sébastien Neukirch \\ Sorbonne Université, UPMC Univ Paris 06, CNRS, UMR 7190 \\ Institut Jean Le Rond d'Alembert,F-75005 Paris, France
}

April 3, 2018

\begin{abstract}
We study an elastic rod bent into an open trefoil knot and clamped at both ends. The question we consider is whether there are stable configurations for which there are no points of self-contact. This idea can be fairly easily replicated with a thin strip of paper, but is more difficult or even impossible with a flexible wire. We search for such configurations within the space of three tuning parameters related to the degrees of freedom in a simple experiment. Mathematically, we show, both within standard Kirchhoff theory as well within an elastic strip theory, that stable and contact-free knotted configurations can be found, and we classify the corresponding parametric regions. Numerical results are complemented with an asymptotic analysis that demonstrates the presence of knots near the doubly-covered ring. In the case of the strip model, quantitative experiments of the region of good knots are also provided to validate the theory.
\end{abstract}

\section{Introduction}

Knots are widely familiar structures, from shoelaces and other everyday use to art forms such as Celtic decoration to the many variants employed by sailors. They are often simple to construct and aesthetically appealing, yet remain topologically and mechanically quite complex. They are also common in biology, appearing in coiled DNA, proteins, and even some species of fish and worms $[41,8]$.

In polymer studies it has been shown that long enough chains are almost always knotted [37], and DNA being a very long polymer various knots arise in linear or circular molecules. DNA knots arise during replication and transcription of the genetic code and the role of enzymes such 


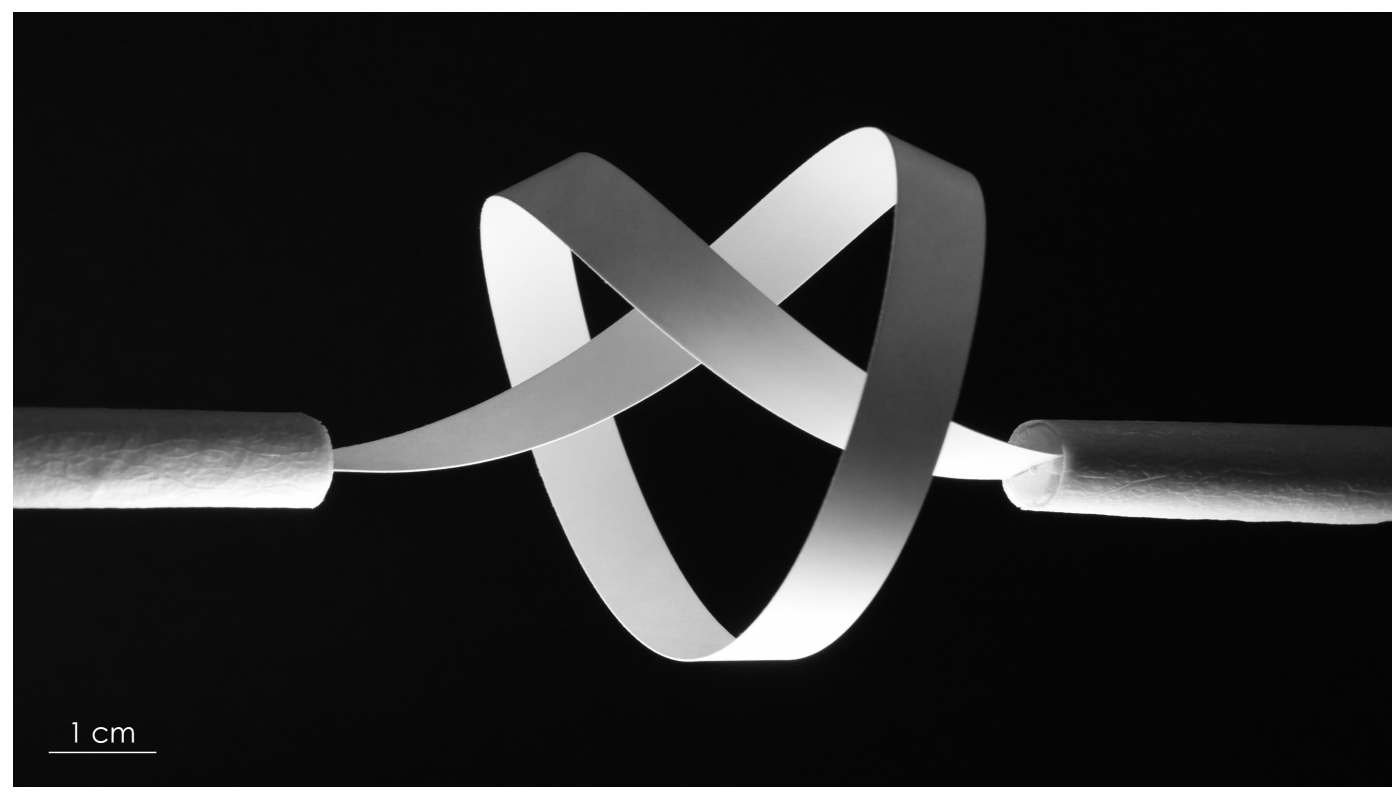

Figure 1: Strip of paper knotted in a trefoil with no self-contact

as topoisomerases is precisely to remove these knots, as the presence of knots has a detrimental effect in the cell and can lead to cell death. In viruses, Arsuaga et al have shown that most of the molecules are knotted due to compaction in the capside [1]. Biological implications of knots in DNA are numerous, e.g. the presence of knots in DNA molecules increases their mobility in gel electrophoresis, the compaction of the chain in knotted regions can prevent transcription, and knots can change the speed with which the virus is ejected from the capside [25]. In this last example, DNA self-contact has been shown to play an important role in the ejection process. In proteins, for many years knotted structures were not detected, but today over 750 knotted configurations are reported in the Protein Data Base; that is more than 1 percent of the entries. These knotted proteins have been shown to catalyse enzymatic reactions [22], play an important role in RNA splicing or in the removing of calcium for the cell by membrane proteins [19], and are also present in plant photoreceptors [40]. Simulations of folding and unfolding of proteins showed that knotted structures have longer unfolding times than unknotted ones [36]. Knots in proteins also have an important effect on their mechanical stability, making them more resistant to degradation. Moreover, the presence of a knot in a structure brings together active-site residues and may promote chemical interactions, drive conformational changes, and drive the spontaneous folding of the structure [18]. The contact interaction in a knotted structure thus appears to be an important feature of its stability and biological activity, and the question of the presence of such contact points or regions in knots therefore emerges.

There are numerous types of questions when studying knots. From a purely mathematical standpoint, in topology a knot is an infinitely thin closed loop, and fundamental issues include knot classification and equivalence of different knot descriptions. A knot in reality obviously differs in that the thickness is finite and the ends are often not closed. This has significant consequences for the mechanician, who is typically interested in the strength, equilibrium shape and dynamic 
behaviour of a knotted filament. Such a description is naturally suited to the theory of elastic rods, but finite thickness in knotted structures by necessity requires consideration of self-contact. Selfcontact poses a significant challenge within a rod theory, and it has been approached in different ways computationally $[7,39,34,33,16,5]$.

While self-contact is an inherent and inevitable feature of tight knots [30], our aim here is to go the other direction. Namely, we consider the question of whether a knotted filament with zero points of self-contact may be realized physically. In particular, our focus is on the simple lab experiment of an elastic rod bent into a trefoil knot, with the ends held clamped (and for simplicity, in the absence of gravity). The shape of such a knot in mechanical equilibrium has been considered before [6,2]. It is characterized by 2 isolated points of self-contact surrounding an interval of self-contact. When twist is increased by rotating one of the clamped ends, this self-contacting solution becomes unstable and the rod jumps to a nearly planar configuration with 4 points of self-contact [6, 5]; see also [20]. The combination of these studies seems to suggest that such an open trefoil will always have points of self-contact in stable equilibrium. Indeed, such a conjecture has been made [21] in the context of a closed knotted elastic rod and extensive numerical computations in the contact-free case have only found unstable knotted configurations [11]. However, it is important to note that the ends of the open knot are taken to be perfectly aligned in the above studies. Experimentally, one can show that if this assumption is removed, then for certain materials and with a little finesse - the right combination of end-rotation and end-displacement - all points of contact can be removed from the open trefoil. We have found that for some materials it is difficult if not impossible to achieve, but the result is fairly easily reproduced with a thin strip of paper or transparency. An example is pictured in Figure 1.

Our objective in this paper is to investigate theoretically such configurations, that is stable equilibrium knots with no points of self-contact. We work within the standard framework of Kirchhoff equations for inextensible, unshearable elastic rods, and determine stability of an equilibrium state via linear stability analysis. To mimic the lab experiment, we model an open rod of finite length clamped with the tangent direction aligned at the ends. The setup for the model is fully outlined in Sec 2. Within this setup, there are three tuning parameters: an end-rotation (related to the linking number), the end-to-end-displacement, and a transverse end-displacement, i.e. an end-shift. The goal is to characterize the structure of the bifurcation space and the possibility for stable contact-free open trefoil knots in terms of these three parameters. To do so, we take two distinct approaches. One is to mimic the paper-strip-in-hands experiment by beginning from a configuration in which the rod is in self-contact, with a fixed end-shift and end-displacement, and then rotating the ends until self-contact is lost and the solution moves to a contact-free state. Varying the end-displacement then enables us to construct an 'island' in parameter space of 'good knots'. This approach forms the subject of Sec 3. The material description of an inextensible rod with diagonal quadratic bending energy can be characterised by two bending parameters. Since our objective is to explore the solution space in terms of the three tuning parameters, in Sec 3 and 6 we restrict to a fixed choice of these two material parameters. However, in modelling a ribbon, for instance a strip of paper as in Fig. 1, the standard constitutive equations in a Kirchhoff theory form at best a rough approximation. Hence, in Sec 4 we take an alternative constitutive description, and using the elastic strip model described in [9] we find similar qualitative behaviour, but a larger overall region of 'good knots'. The predictions within the elastic strip theory are tested 


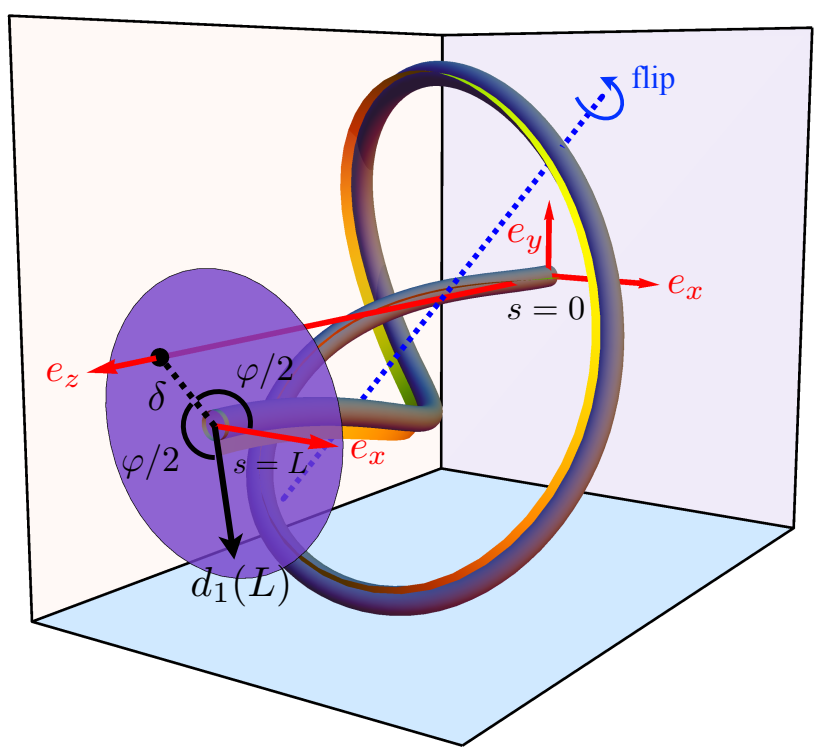

Figure 2: Knotted configuration with end-shift $\delta$ and end-rotation $\varphi$. The director $\mathbf{d}_{\mathbf{1}}$ is materialized through a yellow strip along the rod.

experimentally in Sec. 5, in which thin strips of PVC are clamped to translational and rotational elements in order to quantitatively determine the region of 'good knots'.

As an alternative theoretical approach, we show that knotted structures may be found as perturbations from a doubly covered ring. The stability of such a double ring (ignoring the unphysicality of the continuous self-overlap of the centerline) has been established as a criterion on material parameters [24]. Hence in Sec. 6 we seek knotted configurations in the neighbourhood of the stable double ring, and use the simple analytical form and asymptotic theory to obtain analytical approximations to the region of knots local to the double ring. As well as building an intuitive understanding of the structure of knotted solutions, this analytical approach provides further evidence of the necessity of an end-shift.

Aside from the specialized objective of finding stable knot configurations, in the approaches outlined above we face several computational issues that are more generically applicable, both in terms of numerical computation and asymptotic analysis. Hence, where appropriate, we give a 'recipe' for computation. Finally, conclusions and extensions are discussed in Sec. 7.

\section{Model}

\subsection{Kirchhoff equations}

In this section we outline the governing equations for equilibrium configurations and stability of a Kirchhoff rod. We consider an inextensible, unshearable elastic rod that is naturally straight. We introduce a fixed frame, denoted $\left(\mathbf{e}_{\mathbf{1}}, \mathbf{e}_{\mathbf{2}}, \mathbf{e}_{\mathbf{3}}\right)$, and a continuous family $\left(\mathbf{d}_{\mathbf{1}}(s), \mathbf{d}_{\mathbf{2}}(s), \mathbf{d}_{\mathbf{3}}(s)\right)$ of material frames attached to the cross-sections of the rod, where $s$ is the arc-length along the rod. 
The centerline writes $\mathbf{r}(s, t)=(x, y, z)_{\mathbf{e}_{\mathbf{i}}}$, with $0 \leq s \leq L$ and $L$ the length of the rod. We take the vector $\mathbf{d}_{\mathbf{3}}(s)$ to be aligned with the tangent direction, $\mathbf{r}^{\prime}(s)=\mathbf{d}_{\mathbf{3}}(s)$, where prime denotes differentiation with respect to $s$. The stresses acting at $\mathbf{r}(s, t)$ yield a resultant force $\mathbf{n}(s, t)$ and resultant moment $\mathbf{m}(s, t)$. Note that these quantities can be expressed in either the laboratory frame or the material frame, i.e. we can write $\mathbf{n}=\left(n_{x}, n_{y}, n_{z}\right)_{\mathbf{e}_{\mathbf{i}}}=\left(n_{1}, n_{2}, n_{3}\right)_{\mathbf{d}_{\mathbf{i}}}$. Assuming no rotational inertia, the balance of forces and moments reads:

$$
\begin{gathered}
\text { Balance of forces: } \mathbf{n}^{\prime}+\mathbf{f}=\rho A \ddot{\mathbf{r}} \\
\text { Balance of moments: } \mathbf{m}^{\prime}+\mathbf{d}_{\mathbf{3}} \times \mathbf{n}=0,
\end{gathered}
$$

where $\mathbf{f}(s)$ is a distributed force acting from outside (gravity, contact, etc.), $\rho$ is the density of the material, $A$ the area of the cross-section, and an overdot represents a derivative with respect to time $t$. The material frames being a family of orthonormal frames, their evolution with arc-length $s$ is written with the help of a Darboux vector $\mathbf{u}$

$$
\mathrm{d}_{1}{ }^{\prime}=\mathrm{u} \times \mathrm{d}_{1} \quad, \quad \mathrm{~d}_{\mathbf{2}}{ }^{\prime}=\mathrm{u} \times \mathrm{d}_{2} \quad, \quad \mathrm{~d}_{\mathbf{3}}{ }^{\prime}=\mathrm{u} \times \mathrm{d}_{\mathbf{3}}
$$

which can be interpreted as the strain vector in the rod. We close the set of equations with a constitutive relation between the moment and the strain

$$
m_{i}=\sum_{j=1}^{3} B_{i j} u_{j} \quad \text { for } \quad i=1,2,3
$$

where $\mathbf{B}$ is the rigidities matrix. In this paper, we shall restrict to rods with a diagonal rigidities matrix, for which

$$
\mathbf{m}=B_{11} u_{1} \mathbf{d}_{\mathbf{1}}+B_{22} u_{2} \mathbf{d}_{\mathbf{2}}+B_{33} u_{3} \mathbf{d}_{\mathbf{3}}
$$

\section{Dynamics: equations for components in the material frame}

We write (2.1) using components of $\mathbf{n}$ and $\mathbf{m}$ in the material frames $\left(\mathbf{d}_{\mathbf{1}}(s), \mathbf{d}_{\mathbf{2}}(s), \mathbf{d}_{\mathbf{3}}(s)\right)$. Using (2.2), we have for example $n_{i}^{\prime}=\left(\mathbf{n} \cdot \mathbf{d}_{\mathbf{i}}\right)^{\prime}=\left(\mathbf{n}^{\prime}+\mathbf{n} \times \mathbf{u}\right) \cdot \mathbf{d}_{\mathbf{i}}$ for $i=1,2,3$. The dynamics of the rod then consists of (2.1), (2.2), as well as $\mathbf{r}^{\prime}=\mathbf{d}_{3}$, which forms a set of 18 equations for the 18 variables $\left(x, y, z, n_{1}, n_{2}, n_{3}, m_{1}, m_{2}, m_{3}, d_{1 x}, d_{1 y}, d_{1 z}, d_{2 x}, d_{2 y}, d_{2 z}, d_{3 x}, d_{3 y}, d_{3 z}\right)$, noting that $u_{i}=m_{i} / B_{i i}$ for $i=1,2,3$ via (2.3). These equations are provided in component form in Appendix A.

\section{Non-dimensionalization}

We non-dimensionalize the variables as follows:

$$
\begin{aligned}
& n_{\text {new }}=\frac{n_{\text {old }} L^{2}}{B_{11}}, \quad m_{\text {new }}=\frac{m_{\text {old }} L}{B_{11}}, \quad r_{\text {new }}=\frac{r_{\text {old }}}{L}, \\
& s_{\text {new }}=\frac{s_{\text {old }}}{L}, \quad u_{\text {new }}=u_{\text {old }} L, \quad t_{\text {new }}=\frac{t_{\text {old }}}{L^{2}} \sqrt{\frac{B_{11}}{\rho A}} .
\end{aligned}
$$


In this way, the material properties of the rod are described by two non-dimensional parameters,

$$
K_{2}=\frac{B_{22}}{B_{11}}, \quad K_{3}=\frac{B_{33}}{B_{11}} .
$$

Here, $K_{2}$ is the ratio of bending stiffnesses about $\mathbf{d}_{\mathbf{2}}$ and $\mathbf{d}_{\mathbf{1}}$, while $K_{3}$ is the ratio of torsional stiffness to bending stiffness about $\mathbf{d}_{\mathbf{1}}$. Unless otherwise stated, we will use the fixed values $K=K_{2}=K_{3}=3$ throughout this paper. This choice is motivated by the result in [24] that the double ring is stable if and only if

$$
\sqrt{\frac{K_{2}-1}{K_{2}} \cdot \frac{K_{3}-1}{K_{3}}} \geq \frac{1}{2}
$$

which in the case $K_{2}=K_{3}=K$ reads $K \geq 2$. The choice $K=3$ ensures that the double ring, and at least some neighbourhood around it, is stable. Though we note that these values are already at the limit of being physically attainable in simple cross-sectional geometries (convex, simply connected), as such a rod should satisfy $K \leq 3$ for the Poisson ratio to stay above $-1 / 2$ [23]. For instance, for an elliptical cross-section with major axis $a$ aligned with $\mathbf{d}_{1}$ and minor axis $b$ aligned with $\mathbf{d}_{2}$, one computes [14]

$$
B_{11}=\frac{E \pi a b^{3}}{12}, \quad B_{22}=\frac{E \pi a^{3} b}{12}, \quad B_{33}=\frac{E \pi a^{3} b^{3}}{2(1+\sigma)\left(a^{2}+b^{2}\right)},
$$

where $E$ is the Young's modulus and $\sigma$ the Poisson ratio. The choice $K_{2}=K_{3}=3$ is attained if $a / b=\sqrt{3}$ and $\sigma=-1 / 2$ (auxetic material).

\section{Boundary conditions}

In accordance with the experiment shown in Fig 1, we utilize boundary conditions where the rod is clamped at both ends. We take the $s=0$ end of the rod to be aligned with the fixed frame at all times:

$$
\begin{aligned}
\mathbf{r}(0, t) & =(0,0,0)^{T} & \forall t \\
\mathbf{d}_{\mathbf{1}}(0, t) & =(1,0,0)^{T} & \forall t \\
\mathbf{d}_{\mathbf{2}}(0, t) & =(0,1,0)^{T} & \forall t \\
\mathbf{d}_{\mathbf{3}}(0, t) & =(0,0,1)^{T} & \forall t .
\end{aligned}
$$

while at the $s=1$ end, the rod is held at position $\left(x^{*}, y^{*}, z^{*}\right)$ with its tangent aligned with the $z$ direction

$$
\begin{array}{rr}
\mathbf{r}(1, t)=\left(x^{*}, y^{*}, z^{*}\right)^{T} & \forall t \\
\mathbf{d}_{\mathbf{3}}(1, t)=(0,0,1)^{T} & \forall t .
\end{array}
$$

The final condition is an imposed end-rotation $\varphi$

$$
\begin{aligned}
& \mathbf{d}_{\mathbf{1}}(1, t)=(\cos \varphi, \sin \varphi, 0)^{T} \quad \forall t \\
& \mathbf{d}_{\mathbf{2}}(1, t)=(-\sin \varphi, \cos \varphi, 0)^{T} \quad \forall t .
\end{aligned}
$$


That is, the material vectors $\mathbf{d}_{\mathbf{1}}$ and $\mathbf{d}_{\mathbf{2}}$ lie in the $x$ - $y$ plane at $s=1$, with $\mathbf{d}_{\mathbf{1}}$ making an angle $\varphi$ with the $x$-axis, with $\varphi \in(0,2 \pi)$. In a numerical shooting approach, we thus have 6 equations (2.10a), $d_{3 x}(1, t)=0, d_{3 y}(1, t)=0$, and $\arg \left(d_{1 x}(1, t)+\imath d_{1 y}(1, t)\right)=\varphi$ for the 6 unknowns $\left(n_{1}(0)\right.$, $\left.n_{2}(0), n_{3}(0), m_{1}(0), m_{2}(0), m_{3}(0)\right)$.

\section{Equilibrium}

Setting time derivatives to zero gives an $18 \mathrm{D}$ system to solve for equilibrium configurations (see Appendix A). The boundary conditions read

$$
\begin{aligned}
\mathbf{r}_{\mathbf{e}}(0) & =(0,0,0)^{T} & \mathbf{r}_{\mathbf{e}}(1) & =\left(x^{*}, y^{*}, z^{*}\right)^{T} \\
\mathbf{d}_{\mathbf{1 e}}(0) & =(1,0,0)^{T} & \mathbf{d}_{\mathbf{1 e}}(1) & =(\cos \varphi, \sin \varphi, 0)^{T} \\
\mathbf{d}_{\mathbf{2} \mathbf{e}}(0) & =(0,1,0)^{T} & \mathbf{d}_{\mathbf{2}}(1) & =(-\sin \varphi, \cos \varphi, 0)^{T} \\
\mathbf{d}_{\mathbf{3 e}}(0) & =(0,0,1)^{T} & \mathbf{d}_{\mathbf{3 e}}(1) & =(0,0,1)^{T} .
\end{aligned}
$$

where we denote equilibrium variables with an $e$ index. As stated in the introduction, we wish to consider the rod's configurations in terms of the three 'tuning parameters' of an end-rotation about the tangent, an end-displacement, and an end-shift. The end-rotation is the angle $\varphi$. Since the $s=0$ end is clamped at the origin with the tangent aligned with the $z$-direction in the lab frame, the end-displacement is determined by the value of $z_{e}(1)=z^{*}$, while the end-shift is defined as the magnitude of the distance of the $s=1$ end from the point $\left(0,0, z^{*}\right)$, that is we define

$$
\delta=\sqrt{\left(x^{*}\right)^{2}+\left(y^{*}\right)^{2}}
$$

In the case where $\left(x^{*}, y^{*}\right)=0$ it has been shown that solutions are flip-symmetric $[12,17,27]$, that is the 18 functions $n_{1 e}(s), n_{2 e}(s), \ldots, d_{2 z e}(s), d_{3 z e}(s)$ are either odd or even functions of $s-1 / 2$ and the equilibrium shape of the rod is symmetric through a $\pi$-rotation about the axis passing through point $\mathbf{r}_{\mathbf{e}}(1 / 2)$ and pointing in the direction $\mathbf{d}_{\mathbf{2}}(1 / 2)$. In order to keep the possibility of having flip-symmetric solutions, we choose our transverse end-shift along this flip axis, that is we set

$$
\begin{aligned}
& x^{*}=-\delta \cos (\varphi / 2) \\
& y^{*}=-\delta \sin (\varphi / 2) .
\end{aligned}
$$

Note that this does not prove that shifted solutions will remain flip-symmetric, but allows for the possibility to find such solutions. (A non-flip symmetric solution is shown in Appendix E.)

\section{Stability}

To determine stability of an equilibrium solution, we expand the 18 variables as: $v(s, t)=v_{e}(s)+$ $\epsilon \bar{v}(s) e^{i \omega t}$, e.g. $n_{1}(s, t)=n_{1 e}(s)+\epsilon \bar{n}_{1}(s) e^{i \omega t}$, or $d_{3 x}(s, t)=d_{3 x e}(s)+\epsilon \bar{d}_{3 x}(s) e^{i \omega t}[29,26]$. Inserting these expansions into the dynamic system, Equations (A.1) in Appendix A, while making use of the equilibrium equations (A.2) and only keeping $O(\epsilon)$ terms would give an $18 \mathrm{D}$ eigenvalue problem for the frequency $\omega$. Following [15], we derive a reduced system if, when writing the 
perturbations to the directors $\mathbf{d}_{\mathbf{i}}(s, t)=\mathbf{d}_{\mathbf{i e}}(s)+\epsilon \overline{\mathbf{d}}_{\mathbf{i}}(s) e^{i \omega t}$, we use the equilibrium directors $\mathbf{d}_{\mathbf{i e}}$ to decompose $\overline{\mathbf{d}}_{\mathbf{i}}$, i.e.

$$
\overline{\mathbf{d}}_{\mathbf{i}}(s)=\sum_{j=1}^{3} \alpha_{i j}(s) \mathbf{d}_{\mathbf{j e}}(s) \quad \text { for } \quad i=1,2,3 .
$$

Enforcing orthonormality at order $O(\epsilon)$ yields $\alpha_{i j}=-\alpha_{j i}$ and $\alpha_{i i}=0$. The perturbations to the directors $\overline{\mathbf{d}}_{\mathbf{i}}$ are then determined by only three quantities. We introduce the vector

$$
\boldsymbol{\alpha}(s)=\alpha_{1}(s) \mathbf{d}_{\mathbf{1 e}}+\alpha_{2}(s) \mathbf{d}_{\mathbf{2 e}}+\alpha_{3}(s) \mathbf{d}_{\mathbf{3 e}}
$$

so that $\overline{\mathbf{d}}_{\mathbf{i}}=\boldsymbol{\alpha} \times \mathbf{d}_{\mathbf{i e}}$. Using this expression and $u_{i}(s, t)=u_{i e}(s)+\epsilon \bar{u}_{i}(s) e^{i \omega t}$ for $i=1,2,3$ while expanding (A.1), we obtain differential equations for the $\alpha_{i}(s)$. The equations for the perturbations form a system of only 12 equations, provided in full in Appendix A. The condition that the ends of the rod remain clamped and keep their alignment translates to the requirement that each $\alpha_{i}$ vanishes at the ends. Thus boundary conditions (2.9), (2.10), (2.11), and (2.12) yield

$$
\begin{aligned}
\overline{\mathbf{r}}(0) & =(0,0,0)^{T} & \overline{\mathbf{r}}(1) & =(0,0,0)^{T} \\
\alpha_{1}(0) & =0 & \alpha_{1}(1) & =0 \\
\alpha_{2}(0) & =0 & \alpha_{2}(1) & =0 \\
\alpha_{3}(0) & =0 & \alpha_{3}(1) & =0 .
\end{aligned}
$$

Being conservative, such a system only has real-valued $\omega^{2}$ solutions, and an equilibrium solution is called stable if $\omega^{2}>0$ and unstable if $\omega^{2}<0$. We then have a linear eigenvalue problem for $\omega$, with 12 equations and 12 boundary conditions. Note that when numerically solving this eigenvalue problem, it is convenient to add the normalizing condition

$$
\bar{n}_{1}^{2}(0)+\bar{n}_{2}^{2}(0)+\bar{n}_{3}^{2}(0)+\bar{m}_{1}^{2}(0)+\bar{m}_{2}^{2}(0)+\bar{m}_{3}^{2}(0)=1 .
$$

In a numerical shooting approach, we thus have 7 unknowns $\left(\bar{n}_{i}(0), \bar{m}_{i}(0)\right.$, and $\left.\omega\right)$ and 7 endconditions $\left(\overline{\mathbf{r}}(1)=\mathbf{0}, \alpha_{i}(1)=0\right.$, and Eq. 2.17).

\section{2 'Good knots'}

We have outlined above the mathematical structure governing the equilibrium shape and stability of a clamped elastic rod. As stated, our objective is to seek stable, contact-free, knotted configurations as solutions of this system, in terms of the three tuning parameters $z^{*}$ (end-displacement), $\delta$ (end-shift), and $\varphi$ (end-rotation). To proceed, we must first clarify precisely the configurations we seek. There are three components:

(1) Knotted. A mathematical knot is always a closed curve, its classification being determined by knot invariants. Our interest is the 'simplest' knot, the trefoil. Since we are considering open configurations with clamped ends, we must clarify the definition of a knot in such a geometry. A natural option, employed here, is to create a closed curve by connecting the ends through a giant loop at infinity. Whether the closed configuration forms a 
knot or not depends on how the ends are initially extended beyond the internal portion of the rod (the loop at infinity is irrelevant). Here, we make the simple choice of extending the rod at both ends, $s=0$ and $s=1$, along the tangent direction: we lengthen the rod with 'virtual rays' along the $z$-axis and connect these rays with a loop at infinity.

In most cases, a formal computation of the extension is unnecessary, and classifying the structure as knotted or not is a simple matter, e.g. by visual inspection. While performing parameter continuation, the knotted character is monitored by computing the writhe of the center line of the rod, as self-crossing will make it jump by two units. More precisely, we compute the extended polar writhe $\mathcal{W}_{p}^{\star}$ [31] of the configuration and add to it the total twist $T w=(1 / 2 \pi) \int_{0}^{L} u_{3}(s) \mathrm{d} s$ to obtain a real number that corresponds to the link of the closed curve mentioned earlier, and we focus on configurations with link between 3 and 4 . The use of the extended polar writhe measure enables us to detect unknotting due for example to the Dirac belt trick, see [31, 32], without having to explicitly consider the rays or loop extending the open curve.

We further introduce the definition of a walled configuration. Such a configuration has $0<z(s)<z^{\star}, \forall s \in(0,1)$, that is the rod entirely lies in the space bounded by two walls perpendicular to the $z$-axis. Note that for a walled configuration, the link can be computed using the classical polar writhe [4], as the Dirac belt trick cannot be performed in this case. The walled-knotted configurations are the most clearly identifiable knots visually in a clamped-clamped geometry, and such configurations do not require any concept of tangent extensions. Hence, finding these knots forms a primary focus, though in Section 6 we must relax the walled requirement in order to explore the knotted region asymptotically.

(2) Stable. Stability is the most straightforward component. A configuration is deemed stable if all eigenvalues $\omega$ satisfy $\omega^{2}>0$.

(3) Contact-free. Incorporating self-contact at a finite number of (a priori unknown) points can be achieved within a rod theory $[39,6]$ by introducing force terms with the form of a delta function centered at an unknown $s$-location and with unknown magnitude, both determined by appropriate jump conditions across the contact.

Our approach will primarily be to avoid solutions with contact (with one exception - see Sec 3). In general, rather, we shall work within a contact-free rod theory, with no external forces applied (i.e. $\mathbf{f} \equiv 0$ in (2.1)) and make the assumption that if the centerline of the rod does not self intersect, then the configuration corresponds to a contact-free state. While more care would be required to ensure no contact for a rod with given finite cross-sectional area, this does provide a necessary and sufficient condition for the existence of $a$ rod with no self-contact, as the cross-sectional area can always in theory be decreased, allowing for appropriate increase in Young's modulus to maintain the same bending and twist rigidities.

Under the descriptions above, we seek regions of the $\left(z^{*}-\delta-\varphi\right)$ parameter space corresponding to 'good knots' - walled, knotted, stable, and contact-free configurations. These regions will be bounded by surfaces at which either the configuration becomes unstable $\left(\omega^{2}=0\right)$, does not fit 


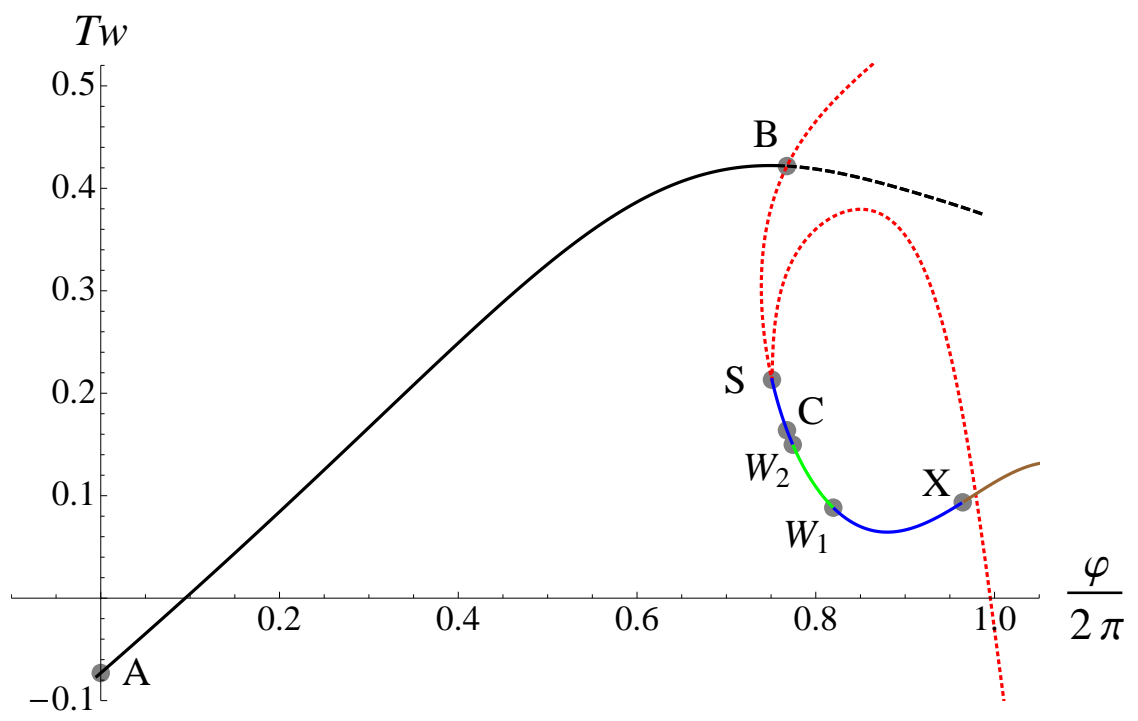

Figure 3: Finding the first good knot. Bifurcation diagram in the $(\varphi / 2 \pi, T w)$ plane for fixed values of $z^{*}=0.115, \delta=0.05$, and $K=3$. At point $A$, the rod is in self-contact (with the ray extensions) at two points. Increasing $\varphi$, experimentally equivalent to rotating the clamped ends, the contact points are lost at point $B$, where the solution jumps to point $C$. A further increase leads to an interval of good knots, between $W_{1}$ and $W_{2}$. A stability boundary is denoted at point $S$, and a self-crossing boundary beyond which the rod is unknotted is denoted point $X$. Corresponding rod configurations at points $A, B$, and $X$ are shown in Figures 4 and 5 .

between two walls $\left(\min _{s \in(0,1)} z(s)=0\right.$ or $\left.\max _{s \in(0,1)} z(s)=z^{*}\right)$, becomes unknotted, or is in selfcontact. The latter two typically correspond to the same thing: self-intersection of the centerline, since a walled knot can only change its topology by passing through itself.

\section{Results in the Kirchhoff rods case}

In this section we compute regions of parameter space corresponding to good knots as numerical solutions of the $18 \mathrm{D}$ system outlined above. A key challenge in this regard is finding a starting point, i.e. a choice of parameters for which a desired configuration is known, and that can provide base values for parametric branch tracing. Below, we show that such a starting point can be attained from a known trefoil shape with contact points by varying the end-rotation $\varphi$ in order to remove the contact points. (Later, in Sec 6, an alternative starting point of an unphysical double ring is employed for an analytical approach.) We fix $K=3$ in the entire Section.

\section{Removing contacts from a trefoil knot}

To start, we fix the parameters $z^{*}=0.115, \delta=0.05$ and consider a rod held with clamps at both extremities, with the clamps consisting of long rigid rays aligned with the $z$-axis. For these values there exists a configuration where the rod contacts the rays at two symmetrical points, one 

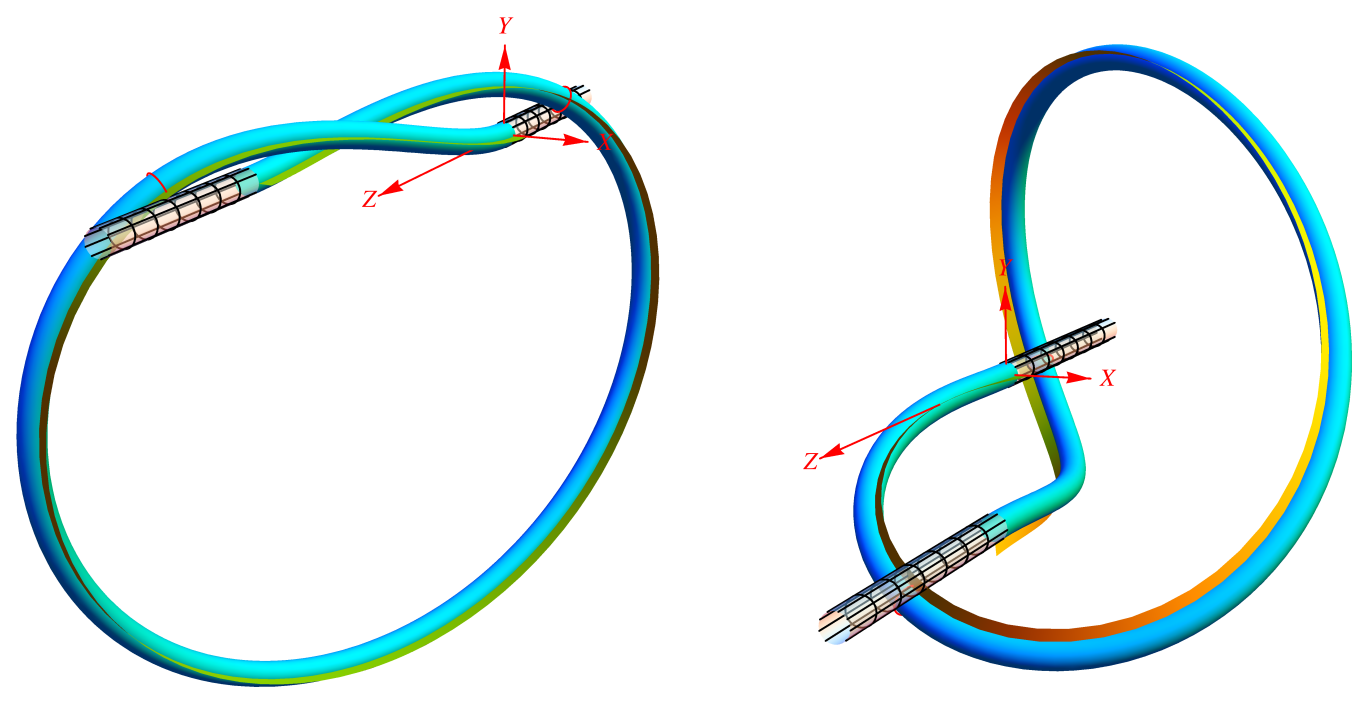

Figure 4: Configurations A and B from Figure 3.

in $z<0$ and the other in $z>z^{*}$, see configuration $A$ in Figure 4. Such a configuration, where the rod is in contact with two obstacles, is obtained by introducing a non-zero shift $\delta=0.05$ on an open trefoil knot with contact [2, 6], following a procedure adapted from [39]. In this configuration $A$, the circular cross-section of the rod (and of the cylindrical rays) has diameter $h=L / 200$ and the contacts happen at $s=0.17$ and $s=0.83$; also the end-rotation is $\varphi=0$, twist $T w=(1 / 2 \pi) \int_{0}^{L} u_{3}(s) \mathrm{d} s \simeq-0.07$, and extended polar writhe $\mathcal{W}_{p}^{\star} \simeq 3.07$. From here, we gradually increase $\varphi$ (experimentally this would mean rotating the $z>z^{*}$ ray around the $z$-axis in a counter-clockwise way) and the corresponding bifurcation diagram is shown in Figure 3 . As $\varphi$ is increased, the intensity of the contact force first increases but then decreases and eventually vanishes when $\varphi \simeq 0.768$, this is configuration $B$ in Figure 3. From this configuration, contact with the rigid rays would require a negative adhesive force, hence the rod jumps to a contact-free knotted configuration, point $C$ in Figure 3. Configuration $C$ is stable and knotted, but not walled as the rod has $z(s)<0$ for $s \in(0.81 ; 0.85)$ and $z>z^{*}=0.015$ for $s \in(0.15 ; 0.19)$. If we further increase the end-rotation from configuration $C$, we find stable walled knots between point $W_{1}$ (with $\varphi_{W_{1}} /(2 \pi) \simeq 0.774$ ) and point $W_{2}$ (with $\varphi_{W_{2}} /(2 \pi) \simeq 0.82$ ). At point $W_{2}$ configurations start to exceed the bounding walls again, though remaining knotted and stable. We eventually arrive at point $X$ (with $\varphi_{X} /(2 \pi) \simeq 0.96$ ) where the rod self-crosses: configurations with $\varphi>\varphi_{X}$ are unknotted.

If from point $C$ we decrease the end-rotation, we see a hysteresis effect: the solution does not jump back to point $B$ but rather follows a branch of knotted, stable, but not-walled solutions. This branch eventually arrives at point $S$ where a pitchfork bifurcation is reached, and stability is lost. Two paths of non flip-symmetric solutions depart from the main path, all three paths being unstable (note that the two paths of non flip-symmetric solutions share the same projection on the $(T w, \varphi)$ plane). The main path (with flip-symmetric solutions) eventually crosses the path of solutions with ray-contact at point $B$. For these parameters $\left(z^{*}=0.115, \delta=0.05, K=3\right)$ 

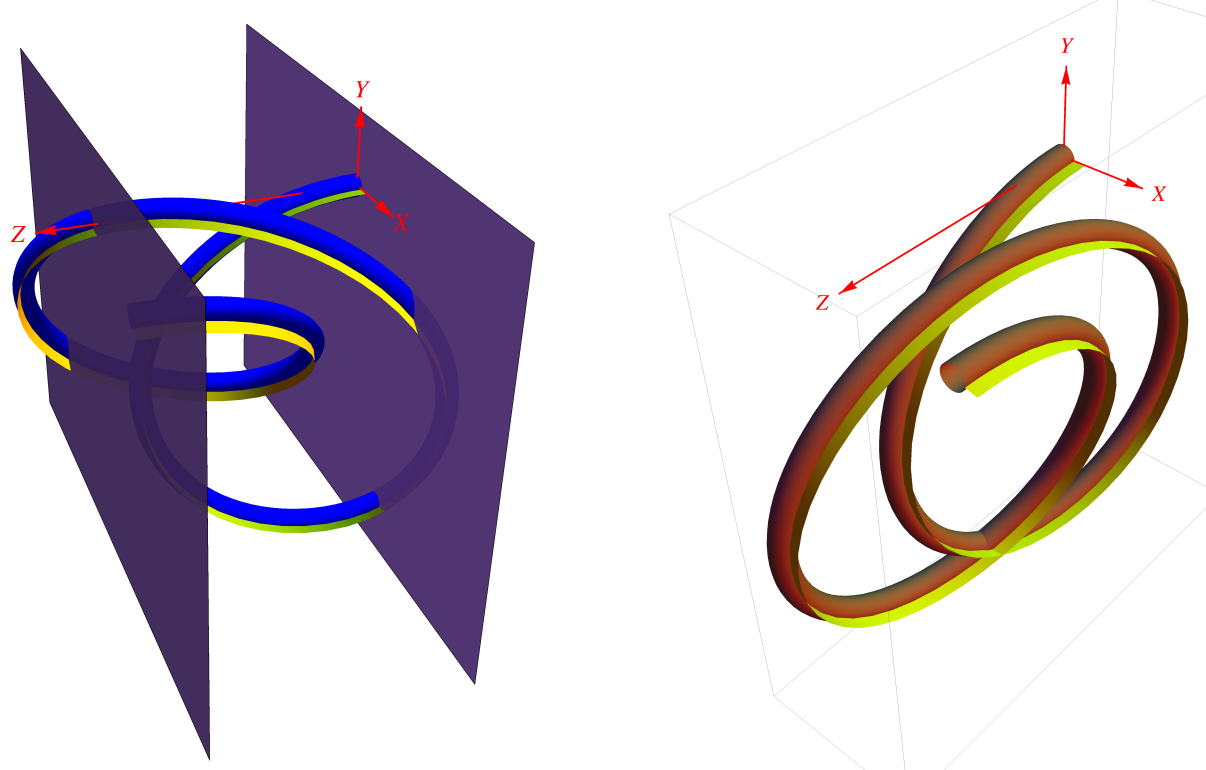

Figure 5: Left: A stable knotted configuration with no self-contact but which does not entirely lie between walls. It belongs to the path between points $W_{1}$ and $X$ in Figure 3 , with $\varphi /(2 \pi) \simeq 0.88$. Right: Configuration $X$ from Figure 3 .
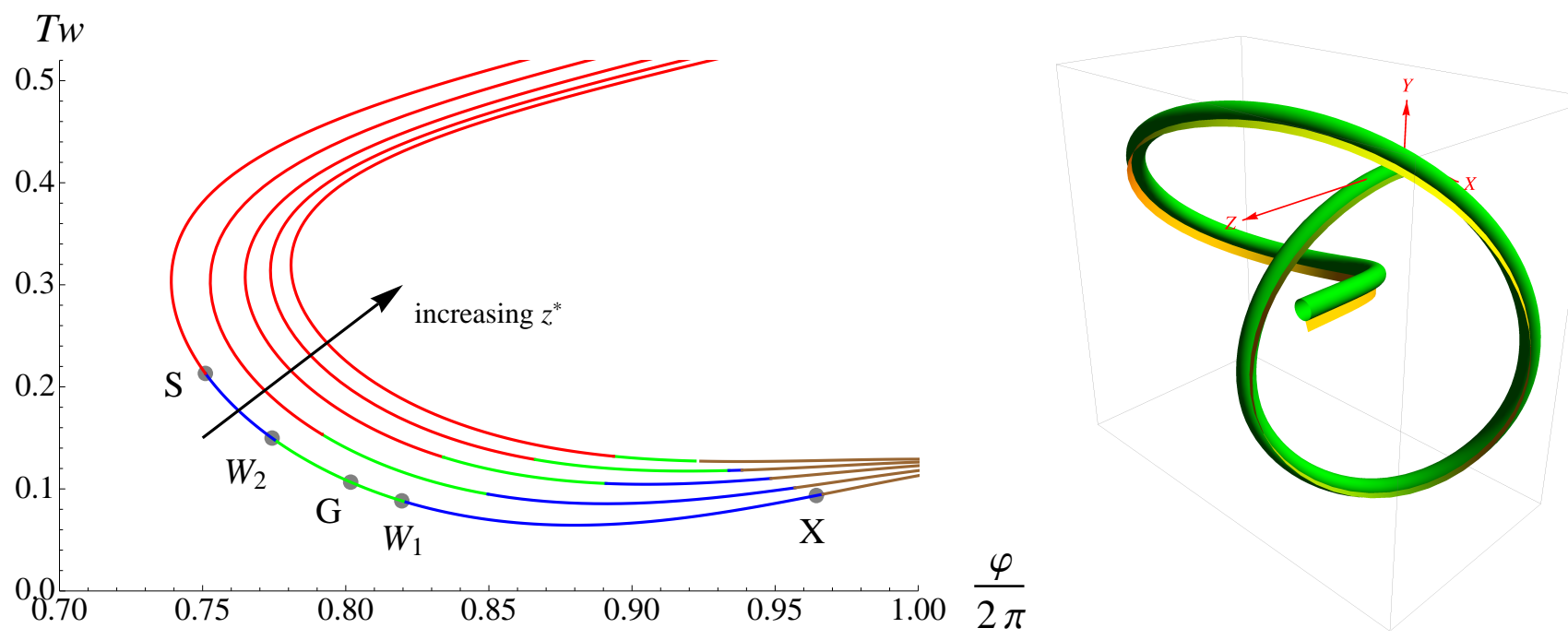

Figure 6: Left: Bifurcation diagrams for different values of end-shortening. Green regions correspond to good knots, blue regions correspond to stable knots that are not between walls, brown regions correspond to unknotted configurations, and red regions correspond to unstable configurations. End-displacement values are $z^{*}=0.115,0.150,0.180,0.200,0.215$. The parameter $\delta=0.05$ is fixed. Right: A stable walled knotted configuration with no self-contact, corresponding to point $G$ on the Left. 

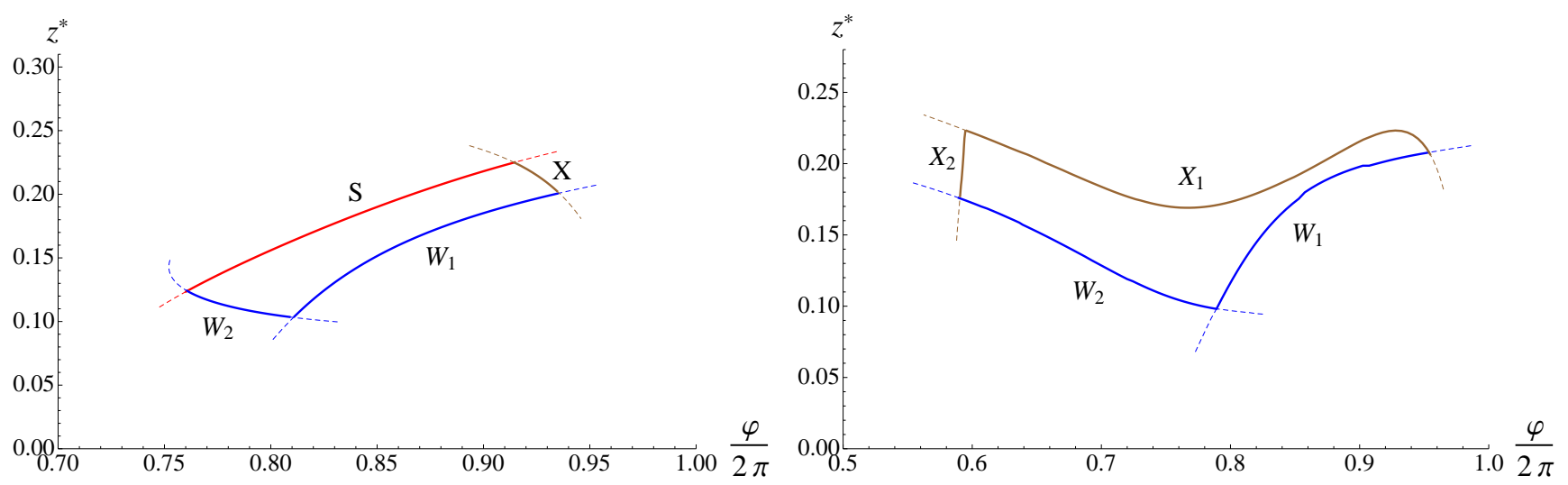

Figure 7: Islands of good knots, plotted in the parameter space of end-displacement $z^{*}$ and endrotation $\varphi$. The end-shift is fixed at $\delta=0.05$. Left: The Kirchhoff rod case, where good knots lie inside the region limited by the four curves $S, W_{1}, W_{2}$, and $X$. Right: The elastic strip case, where good knots lie inside the region limited by the four curves $W_{1}, W_{2}, X_{1}$, and $X_{2}$.

we see that non-flip symmetric knots are all unstable. For other parameter values good non-flip symmetric knots have been found, though here we will not investigate further non-flip symmetric solutions.

\section{Island of 'good knots'}

The computations above confirm the theoretical presence of stable, walled, and contact-free knots, indeed showing a continuous interval of such 'good knots'. We now allow the end-displacement $z^{*}$ to vary, while keeping $\delta=0.05$; the result appears in Figure 6-left, which plots paths of flipsymmetric solutions for different $z^{*}$ values. Here, as in Fig 3, green regions represent good knots, blue regions are stable knots that are not between walls, brown regions are unknotted, and red regions are unstable. We see that the interval of end-rotation values for which good knots exist is drifting slowly with $z^{*}$. We therefore plot in Figure 7-left a connected set of values of $\varphi$ and $z^{*}$ for which these stable walled knots exist, i.e. a parametric island of good knots. As illustrated in Fig 7-left, there are three ways for a 'good' configuration to lose its properties: $(i)$ it may go unstable (cross the $S$ curve), ( $i i)$ it may cross itself and unknot (the $X$ curve), or (iii) it may extend outside the $z=0$ and $z=z^{*}$ bounding walls (the $W_{i}$ curves). Our numerical computations suggest that this island of good knots shrinks as $\delta$ is decreased and vanishes completely for a finite value of $\delta$, implying that an end-shift is a requirement for a good knot. Further evidence for this hypothesis is provided by asymptotic calculations for small $\delta$ in Section 6 .

\section{Results in the elastic strip case}

One of the assumptions for the validity of Kirchhoff rod theory is that the cross-section is small compared to the total length of the structure. Moreover the aspect ratio of the cross-section itself should not be too large. For a strip of paper, as in Fig 1, the rectangular cross-section, of width $w$ 
and thickness $h$, is flat, $h \ll w$, so the assumptions for Kirchhoff theory are not met. In such a case, the system should be modelled using elastic strip equations [13, 35]. Here we follow the approach developed in [10], where elastic strip equations are derived using an energy approach starting from shell theory. It is shown that in the regime where $L \gg w \gg h$, an elastic strip obeys essentially the same equations as an elastic rod, provided the bending and twist constitutive relations are changed in the following way. We put the width $w$ along the $\mathbf{d}_{\mathbf{1}}$ direction and the thickness $h$ along the $\mathbf{d}_{\mathbf{2}}$ direction. As a consequence the strip is unbendable around $\mathbf{d}_{\mathbf{2}}$ and we thus have

$$
u_{2}(s, t)=0 \quad \forall(s, t)
$$

even if the bending moment $m_{2}$ is non-zero. This situation is analogous to the constraint of inextensibility where the extensional strain in the rod is zero while the axial stress is not. A second constraint, coming from differential geometry, is that the inextensibility of the strip surface itself implies a link between the bending in the soft direction $\left(u_{1}\right)$ and the twist $\left(u_{3}\right)$, eventually modifying the other two constitutive relations to

$$
\begin{aligned}
& m_{1}=B\left(1-\frac{u_{3}^{4}}{u_{1}^{4}}\right) u_{1} \\
& m_{3}=2 B\left(1+\frac{u_{3}^{2}}{u_{1}^{2}}\right) u_{3}
\end{aligned}
$$

where $B=(1 / 12) h^{3} w /\left(1-\nu^{2}\right)$, with $\nu$ the Poisson's ratio of the material. In the following, we use $B$ instead of $B_{11}$ to non-dimensionalize physical quantities, see (2.5).

The dynamics of the strip is then given by system (A.1), complemented by (4.1) and (4.2): a differential-algebraic system that we will try to avoid. In principle (4.2) could be inverted so as to express $u_{1}$ and $u_{3}$ as functions of $m_{1}$ and $m_{3}$ and one could treat the system (A.1) as was done for the rod case: a system of 18 differential equations for the 18 variables $\left(x, y, z, n_{1}, n_{2}, n_{3}\right.$, $\left.m_{1}, m_{2}, m_{3}, d_{1 x}, d_{1 y}, d_{1 z}, d_{2 x}, d_{2 y}, d_{2 z}, d_{3 x}, d_{3 y}, d_{3 z}\right)$. Nevertheless the inversion leads to multiple roots, a matter that complicates the approach. We therefore use an alternative procedure and differentiate (4.2) with respect to $s$ in order to write

$$
\begin{aligned}
& u_{1}^{\prime}=\frac{u_{1}^{3}}{\left(u_{1}^{2}+u_{3}^{2}\right)^{3}}\left[u_{1}\left(u_{1}^{2}+3 u_{3}^{2}\right) m_{1}^{\prime}+2 u_{3}^{2} m_{3}^{\prime}\right] \\
& u_{3}^{\prime}=\frac{u_{1}^{2}}{2\left(u_{1}^{2}+u_{3}^{2}\right)^{3}}\left[\left(u_{1}^{4}+3 u_{3}^{4}\right) m_{3}^{\prime}+4 u_{1} u_{3}^{3} m_{1}^{\prime}\right],
\end{aligned}
$$

where $m_{1}^{\prime}$ and $m_{3}^{\prime}$ are replaced using (A.1d), (A.1f), and (4.1). System (A.1), (4.3) then comprises 20 equations for the 20 variables $\left(x, y, z, n_{1}, n_{2}, n_{3}, m_{1}, m_{2}, m_{3}, d_{1 x}, d_{1 y}, d_{1 z}, d_{2 x}, d_{2 y}\right.$, $\left.d_{2 z}, d_{3 x}, d_{3 y}, d_{3 z}, u_{1}, u_{3}\right)$ with boundary conditions given by (2.9), (2.10), and (2.11), supplemented with (4.2) evaluated at $s=0$. Numerical shooting now involves the 6 unknowns $\left(n_{1}(0)\right.$, $\left.n_{2}(0), n_{3}(0), u_{1}(0), m_{2}(0), u_{3}(0)\right)$ and the 6 equations $(2.10), d_{3 x}(1, t)=0, d_{3 y}(1, t)=0$, and $\arg \left(d_{1 x}(1, t)+\imath d_{1 y}(1, t)\right)=\varphi$. If one interprets the non-linear constitutive relation (4.2a) as a linear relation such that the term in parentheses plays the role of a non-uniform effective bending rigidity, one sees that the local twist rate $u_{3}(s)$ lowers the rigidity in bending. This effective rigidity 

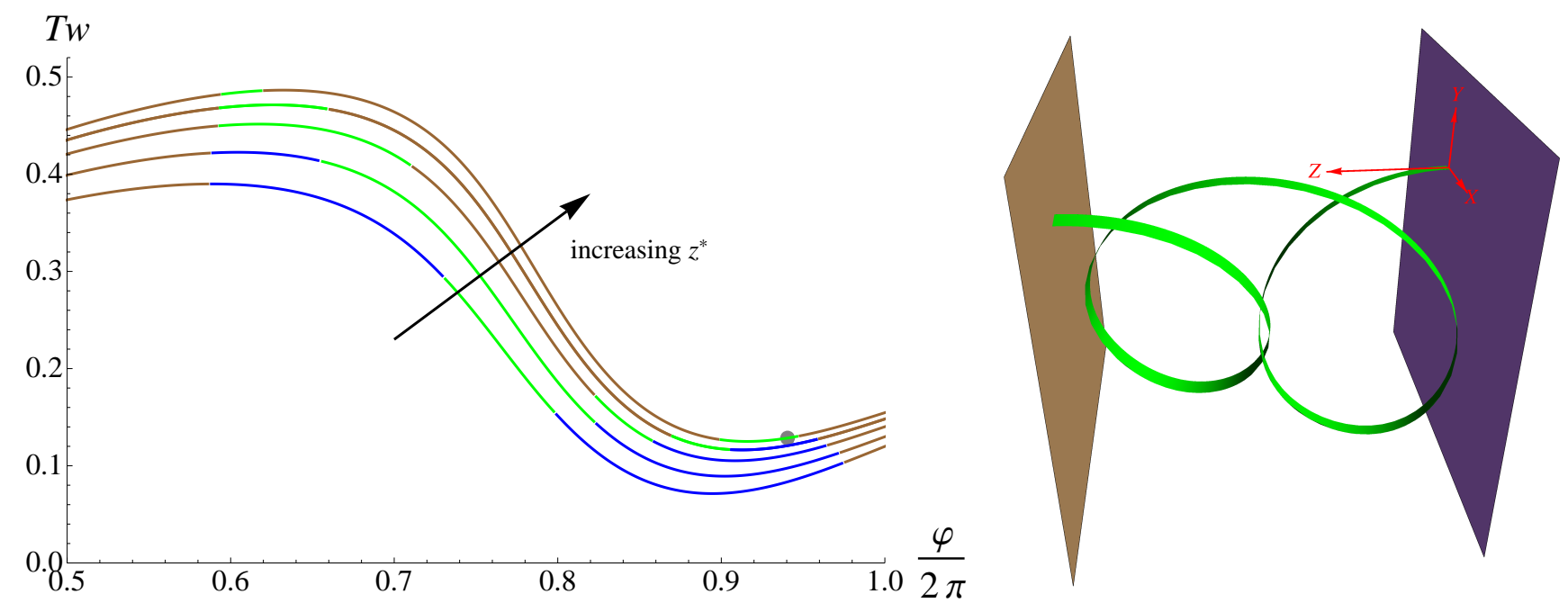

Figure 8: Left: Bifurcation diagram for different values of the end-displacement $z^{*}$ in the elastic strip case. Green regions represent good knots, blue regions stable knots that are not between walls, and brown regions unknotted configurations. End-displacement values are (following the arrow) $z^{*}=0.115,0.150,0.180,0.200,0.215$. The parameter $\delta=0.05$ is fixed. The point on the upper curve corresponds to the configuration shown on the right. Right: A stable walled knotted elastic strip configuration with no self-contact, with $z^{*}=0.215$ and $\varphi /(2 \pi) \simeq 0.94$.

is cancelled at those points along the rod where $u_{3}(s)=u_{1}(s)$ and reversed when $\left|u_{3}(s)\right|>\left|u_{1}(s)\right|$. The relation $(4.2 \mathrm{~b})$ shows that the opposite is true for the twist constitutive relation: the local twist rate increases the effective twist rigidity.

\section{Island of 'good knots' in the elastic strip case}

As with the Kirchhoff model, we compute regions of the parameter space corresponding to good knots in the elastic strip case. In Figure 8, a bifurcation diagram in the $(T w, \varphi)$ projection is shown for the same values of $z^{*}$ and $\delta$ as in Figure 6-left. We see that the end-rotation range for which knotted but not walled (blue curves) and good knot (green curves) configurations exist is larger in the strip case than in the rod case. Moreover, equilibrium configurations are found down to values $\varphi<0.5$, and no pitchfork bifurcation nor non-flip-symmetric solutions are found. In comparison with the Kirchhoff rod case, we plot in Figure 7-right the island of good knots for the strip model, i.e. the parameter range $\left(\varphi, z^{*}\right)$ corresponding to good knots, keeping the end-shift fixed at $\delta=0.05$. In the 3D parameter space $\left(\delta, z^{*}, \varphi\right)$, we have not found any good knots outside the region bounded by $0.01<\delta<0.3,0.05<z^{*}<0.45$, and $0.02<1-\varphi /(2 \pi)<0.5$, see Figure 9. Nevertheless, as in the Kirchhoff rod case, we have numerically found stable knotted unwalled configurations as $\delta \rightarrow 0$, see Section 6 for asymptotic calculations on this matter. 


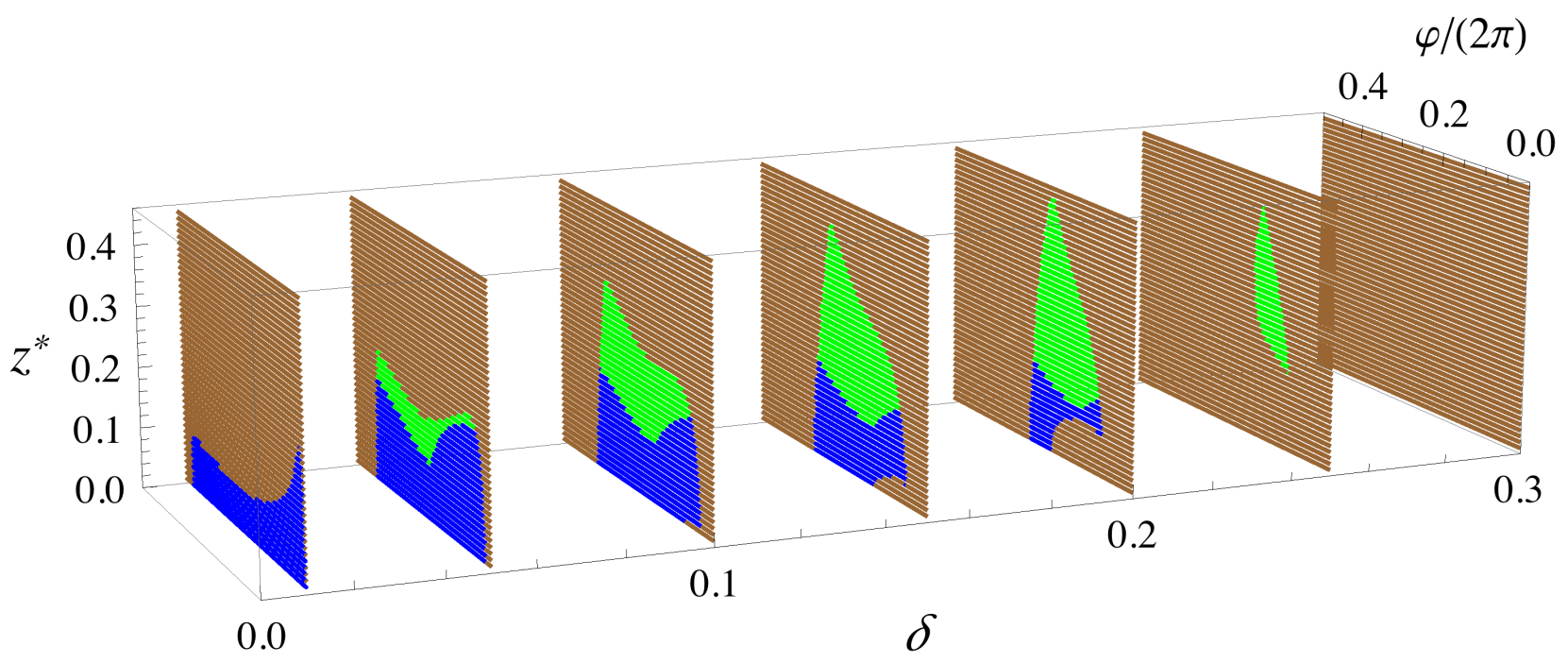

Figure 9: In the elastic strip case, regions for good knots (green), unwalled knots (blue), and unkotted configurations (brown) in the $3 \mathrm{D}$ space $\left(\delta, z^{*}, \varphi\right)$, for $\delta=0.01,0.05,0.10,0.15,0.20$, 0.25 , and 0.30 .

\section{Experimental validation}

We have shown that parameter values exist for stable, contact-free and walled knots, in both the Kirchhoff rod and Elastic Strip cases. As highlighted in the introduction, the three parameters of end-displacement, end-rotation, and end-shift are easily manipulated in an experimental setting. In this section, we perform a quantitative experiment to test and validate the theoretical predictions. In particular, we focus experimentally on the elastic strip case. As seen above, the strip model predicts a larger region of good knots, thus increasing the chances of finding these configurations experimentally. Also, a material fitting the assumptions of the strip model is easily obtained, while the material parameters we have utilised in the Kirchhoff theory $\left(K_{2}=K_{3}=3\right)$, though physically possible, are much harder to manufacture (potentially requiring an auxetic material).

\section{Experimental setup}

The experimental setup is presented in Figure 10-left. We use strips cut from PVC binding cover, with length $L=26.3 \mathrm{~cm}$, width $w \in(6,15) \mathrm{mm}$, thickness $h=200 \mu \mathrm{m}$, Young's modulus $E \simeq 3$ GPa, and density $\rho=1380 \mathrm{~kg} / \mathrm{m}^{3}$. At both ends clamps, laser-cut from a $8 \mathrm{~mm}$ thick PMMA sheet, bind the strip to rotational Thorlabs elements (enabling precise tuning of $\varphi$ ), and these elements themselves are mounted on Thorlabs translational elements, one in the $z$ direction for tuning end-displacement $z^{\star}$ and one in the transverse $x$ direction, for $\delta$ tuning. Translational precision is around $1 \mathrm{~mm}$, thus the error for $z^{\star}$ and $\delta$ is around $\pm 0.2 \%$. For rotation, the imprecision in angle is around $1^{\circ}$, so the error on the link $\varphi$ is around $\pm 0.3 \%$. For a given strip, the experimental procedure is to fix the end-shift and the end-rotation, and then vary the end-displacement (by sliding one rotational clamp along its translational axis in the $z$-direction) until self-contact or 

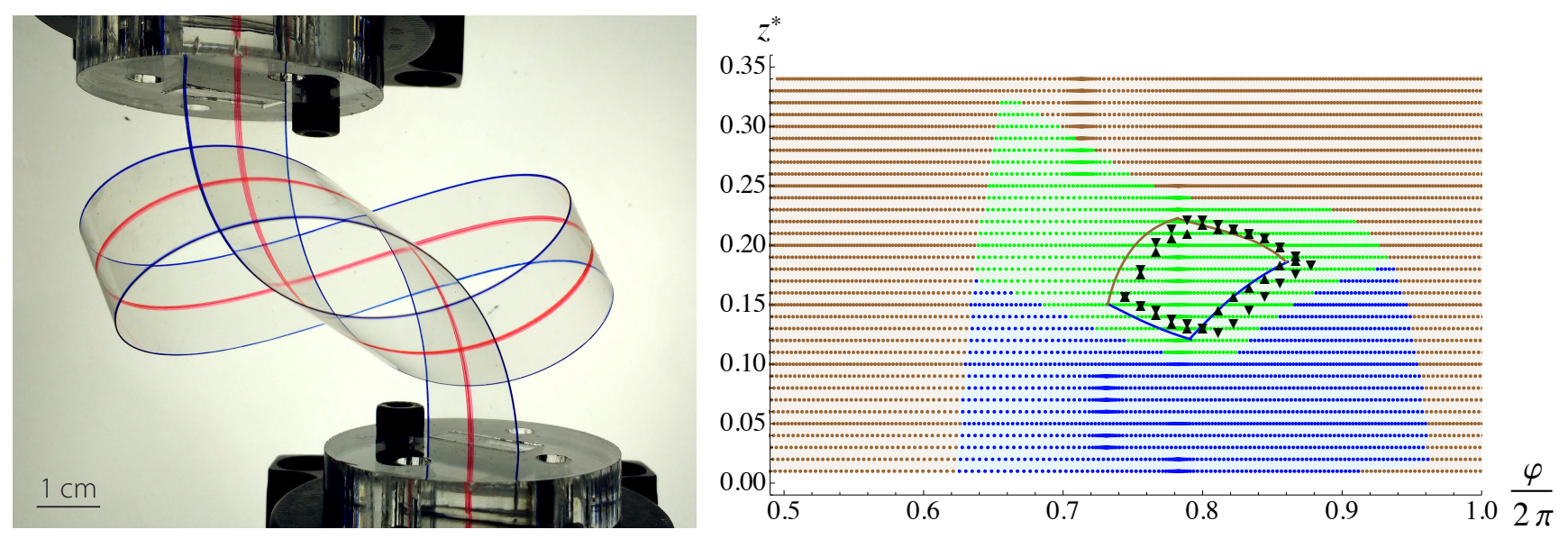

Figure 10: Left: Picture of a good knot for a PVC strip of width $w / L=0.057$. Here $z^{\star}=0.17$, $\varphi /(2 \pi)=0.78$ and $\delta=0.10$. Right: The island of the good knots for the strip model: Comparison between theoretical predictions and experimental results. Colored regions are computed in the limit $w=0$, while the continuous lines shows boundaries once the finite thickness $w / L=0.057$ is taken into account. Experimental points correspond to boundaries found with strips facing up (upward triangles) and down (downward triangles) in the gravity field. The end-shift is $\delta=0.10$.

wall contact occurs, if possible. In this way any values of end-to-end displacement that enables a good knot are precisely determined. The end-rotation is then varied slightly and the procedure is repeated. Regions of good knots could indeed be located. A comparison between theory and experiment is provided in Figure 10-right for an end-shift $\delta=0.10$ and $w=15 \mathrm{~mm}$. Here, the colored background is the numerical output from the strip model, the green region representing good knots. However, we note that in the model calculations, the self-crossing boundary was computed according to self-intersection of the centreline (as if $w=0$ ). To compare with the present experiment, we adapt the numerics in order to take into account the finite width of the strip: the solid curves show the updated boundaries, here the appreciable width causes the region of good knots to be significantly smaller. The triangle points represent experimentally determined boundaries of the region of good knots for 'up' and 'down' orientations of the strip in the gravity field. Gravity has a clear effect experimentally (note that $L^{3} \rho g h w / E I \simeq 25$ ) and the size of the good region varies with the orientation of the strip. A good knot in the down configuration is presented in Figure 1 where the major loop points downwards. The up configuration, on the other hand, corresponds to the case where the major loop points upwards. While there is some discrepancy, generally the agreement is good. We also note that small plastification was present: when removing the strip from the clamps, it would show a slight intrinsic curvature (end deflection of about $5 \%$ of the total strip length).

\section{Validity of the strip model : importance of the ratio $w / h$}

Experiments were conducted for several different strip widths. In all experiments, the crosssectional thickness of PVC used was $h=200 \mu \mathrm{m}$, and we varied the width from $w \approx 6 \mathrm{~mm}$ to 
$w \approx 15 \mathrm{~mm}$, hence the aspect ratio $w / h$ varied from about 30 to 75 . The behaviour of the system was found to depend on the width $w$ of the strip as follows:

- For $w$ lower than $\sim 6 \mathrm{~mm}(w / h<30)$, the presence of dynamic instabilities was observed, reminiscent of the Kirchhoff rod case: at particular fixed $z^{\star}$, decreasing $\varphi$ slowly would lead to a sudden jump to a new configuration.

- For $w$ larger than $\sim 6 \mathrm{~mm}(w / h>30)$, stability jumps were not observed, though we found that the size of the 'good knot' island was strongly dependent on $w$. Moreover, for $w \lesssim 8$ $\mathrm{mm}(w / h=40)$, experimental results did not match well with predictions of the elastic strip model. Improvement was observed for a wider strip $w=10 \mathrm{~mm}$ but only for $w \gtrsim 15 \mathrm{~mm}$ did the comparison between theoretical prediction and experimental results become satisfactory.

This $w / h$ dependence is not included in the elastic strip model, which treats the strip as a plate with no extension, that is $u_{2}$ is constrained to be zero at all times, an assumption that is met experimentally for large $w / h$ ratios only. As this ratio becomes smaller, say $O(10)$, the curvature $u_{2}$ is no longer infinitely small compared to $u_{1}$ and $u_{3}$ and the elastic strip model should then be enriched in order to describe such experimental systems.

\section{Unfolding of the doubly covered ring}

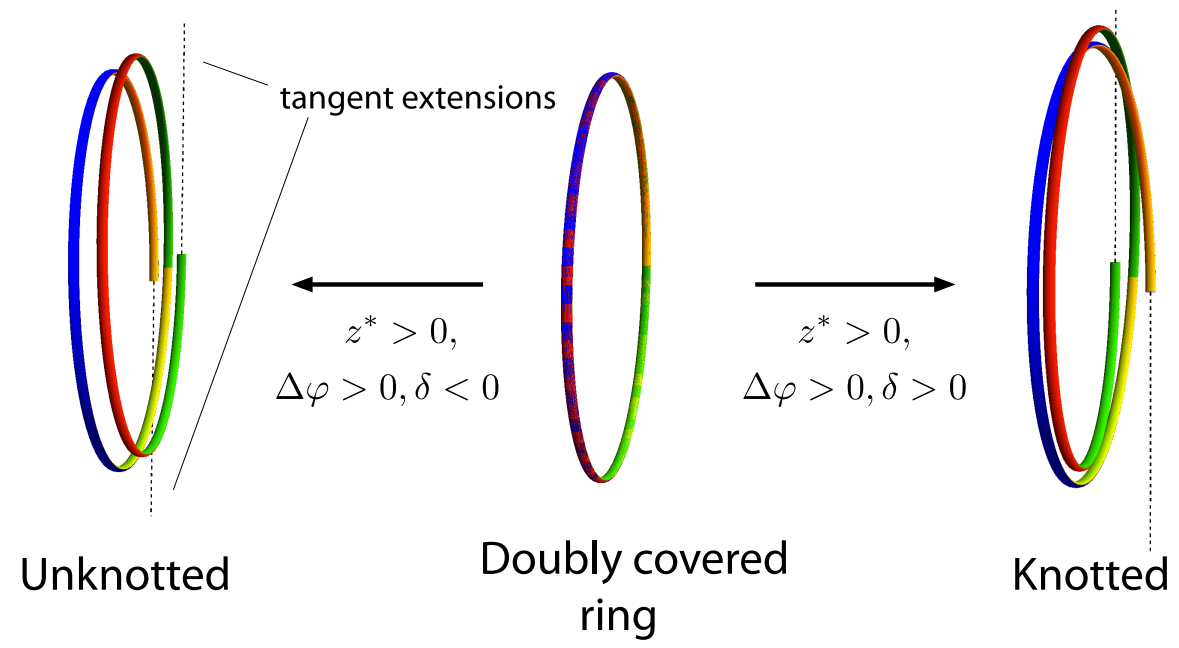

Figure 11: Beginning at the the doubly-covered ring (middle) and adding a small end-displacement, a small end-rotation and end-shift of opposite sign creates an unknotted helical-type configuration (left), while a small end-rotation and end-shift of equal sign can create a knotted configuration (right).

As an alternative approach to finding regions of parameter space with 'good knots', in this section we take as a starting point the doubly covered ring - this is an unphysical configuration 


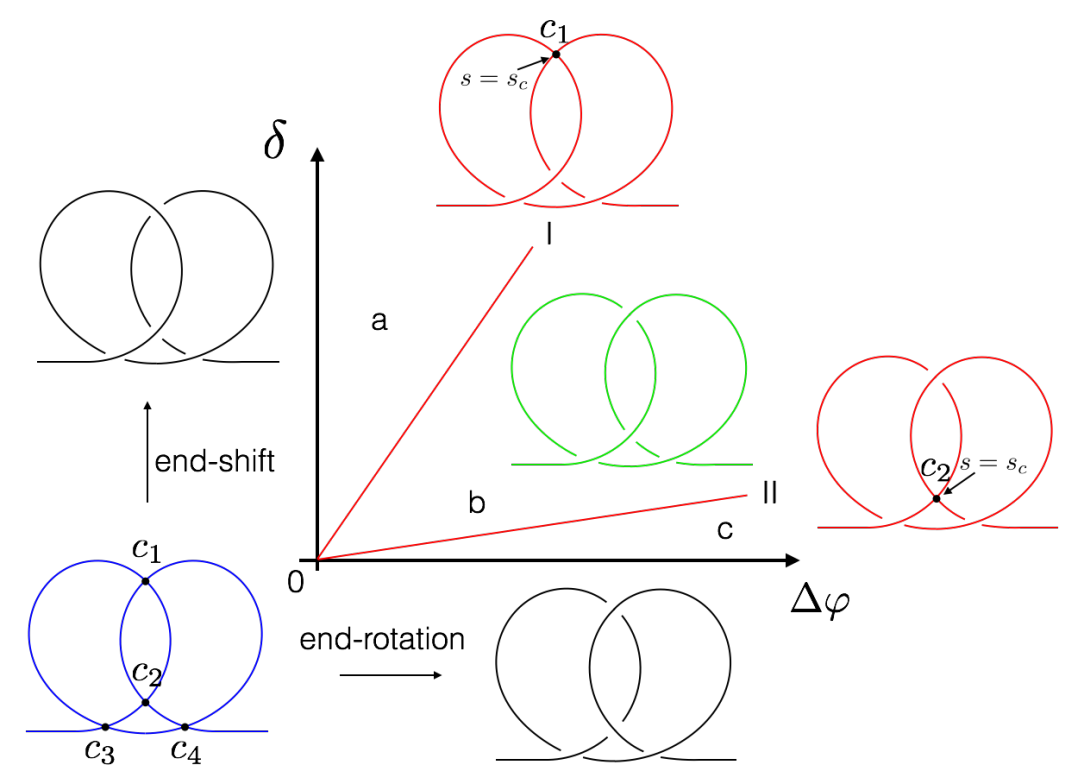

Figure 12: Schematic of configurations of rod centerline for end-displacement $0<z^{*} \ll 1$. The origin (which corresponds to zero end-rotation $(\Delta \varphi=0)$ and zero end-shift $(\delta=0)$ has 4 points of self-contact. Small changes in $\delta$ and $\Delta \varphi$ can remove the self-contact and divide the quadrant $\delta>0, \Delta \varphi>0$ into 3 regions, separated by two curves with one point of self-contact each. Knotted solutions exist only in the wedge region (b).

in which the centerline is a circle that traces over itself exactly once and that corresponds to end-rotation $\varphi=2 \pi$, end-shift $\delta=0$, and end-displacement $z^{*}=0$. The key observation is that knots can be found emerging from this configuration upon application of a small tuning of the parameters. This idea is easily replicated with a strip of paper: curve a thin strip of paper into a double circle, such that the ends are in contact, and then shift the ends in the transverse direction. Shifting in one direction creates an unknotted helical-type shape, while passing one end through the middle and shifting in the other direction creates a knot, as illustrated in Figure 11. Here we have included the tangent extensions to the numerically computed configurations, from which it can be concluded that the configuration on the right is indeed a knot. We note that in these configurations, with the ends very close, we cannot hope to have a knot contained between walls, so this requirement is removed for this section.

We work with the Elastic Strip model where the doubly covered ring is stable [3] and consider nearby knotted equilibrium configurations. It is reasonable to expect that these knotted configurations will be stable and our approach in this section is to seek such a region of stable knots by means of an asymptotic expansion about the doubly-covered ring, i.e. in the limit $0<z^{*} \ll 1$, $0<\Delta \varphi \ll 1,0<\delta \ll 1$, with $\Delta \varphi=2 \pi-\varphi$. In particular, our objective is to uncover analytically the intuitive picture shown schematically in Figure 12. For small but positive $z^{*}$, if there is zero end-rotation and zero end-shift $(\Delta \varphi=\delta=0)$, the centreline is a planar double loop configuration with 4 points of self-contact, labelled $c_{i}, i=1 \ldots 4$. From there, applying either a small end shift or end-rotation gives an unknotted state with no points of contact. To obtain a knotted state, the loops must cross, i.e. the centerline must pass through itself at $c_{1}$ or $c_{2}$. Applying both a 
small end-shift and end-rotation divides the positive quadrant of the $(\delta-\Delta \varphi)$ plane into 3 distinct regions, bounded by two curves (labelled I and II) with a single point of self-contact $\left(c_{1}\right.$ and $c_{2}$, respectively). Within the wedge region between these curves, the loops are crossed and the centreline curve is knotted. We seek expressions for these boundary curves.

To make analytical progress, we proceed entirely within the assumption of flip-symmetry and calculate equilibrium solutions without assessing stability, as stability is already established for the base solution of the double ring and nearby solutions should then also be stable. Nevertheless we have verified numerically that the solutions attained below are stable. For the purposes of the present analysis, we also make several notational choices for convenience. First, for symmetric solutions, it is convenient to shift the domain of $s$ to be $s \in(-1 / 2,1 / 2)$ and to place the origin at the midpoint $s=0, \boldsymbol{r}(0)=\mathbf{0}$. Second, we rotate the fixed frame so that at the mid-point the rod is oriented such that

$$
\mathbf{d}_{\mathbf{1}}(0)=\left(\begin{array}{c}
\cos \xi \\
0 \\
-\sin \xi
\end{array}\right), \quad \mathbf{d}_{\mathbf{2}}(0)=\left(\begin{array}{l}
0 \\
1 \\
0
\end{array}\right), \quad \mathbf{d}_{\mathbf{3}}(0)=\left(\begin{array}{c}
\sin \xi \\
0 \\
\cos \xi
\end{array}\right) .
$$

In this orientation, the flip-symmetry implies $n_{2}(0)=n_{y}(0)=0$ and $m_{2}(0)=m_{y}(0)=0$, while the remaining components of $\mathbf{n}$ and $\mathbf{m}$ are unknown and must be determined as part of the asymptotic solution. We have therefore 9 unknowns $n_{x}(0), n_{z}(0), m_{x}(0), m_{z}(0), \xi, z^{*}, \Delta \varphi$, $\delta$, and $s_{c}$, the latter being the arc-length of the self-contact point. The end-rod boundary conditions in (2.12) are then written as

$$
d_{3 x}(1 / 2)=0, d_{3 y}(1 / 2)=0, x(1 / 2)=\delta / 2, z(1 / 2)=z^{*} / 2, d_{1 y}(1 / 2)=-\sin (\Delta \varphi / 2),
$$

and the contact conditions read

$$
x\left(s_{c}\right)=0, z\left(s_{c}\right)=0 .
$$

We have 7 conditions for 9 unknowns, and we look at the solution of this boundary value problem in the form $\delta=\delta\left(z^{*}, \varphi\right)$, that is we set $z^{*}=\epsilon$ and $\Delta \varphi=\beta \epsilon$ and look for the solution as a series expansion in $\epsilon$, with $0<\epsilon \ll 1$, and $\beta$ fixed. We expand all the variables $\mathbf{r}, \mathbf{n}, \mathbf{m}, \mathbf{u}, \mathbf{d}_{\mathbf{i}}, \xi, \delta$, and $s_{c}$ in powers of $\epsilon$ up to order 3. For example we write

$$
\begin{aligned}
\mathbf{r}(s) & =\mathbf{r}^{[0]}(s)+\epsilon \mathbf{r}^{[1]}(s)+\epsilon^{2} \mathbf{r}^{[2]}(s)+\epsilon^{3} \mathbf{r}^{[3]}(s)+O\left(\epsilon^{4}\right) \text { or } \\
\mathbf{m}(s) & =\mathbf{m}^{[0]}(s)+\epsilon \mathbf{m}^{[1]}(s)+\epsilon^{2} \mathbf{m}^{[2]}(s)+\epsilon^{3} \mathbf{m}^{[3]}(s)+O\left(\epsilon^{4}\right)
\end{aligned}
$$

At zeroth order, the doubly covered ring solution is

$$
x^{[0]}(s)=0, y^{[0]}(s)=\frac{\cos (4 \pi s)-1}{4 \pi}, z^{[0]}(s)=\frac{\sin (4 \pi s)}{4 \pi}
$$

and, following [6], we use a rotating frame to write the component of the unknown vectors $\mathbf{n}, \mathbf{m}$, $\mathbf{u}, \mathbf{d}_{\mathbf{1}}, \mathbf{d}_{\mathbf{2}}$, and $\mathbf{d}_{\mathbf{3}}$. The rotating frame is constructed from the Frenet frame of the curve (6.6) and coincides with the fixed frame at $s=0$.

$$
\mathbf{e}_{\mathbf{x}}=\left(\begin{array}{l}
1 \\
0 \\
0
\end{array}\right), \quad \mathbf{e}_{\mathbf{t}}=\left(\begin{array}{c}
0 \\
\cos 4 \pi s \\
\sin 4 \pi s
\end{array}\right), \quad \mathbf{e}_{\tau}=\left(\begin{array}{c}
0 \\
-\sin 4 \pi s \\
\cos 4 \pi s
\end{array}\right) .
$$


Furthermore we use $(X, Z, X)$ Euler angles $(\psi, \theta, \phi)$, see Appendix B, to describe the rotation of the material frame and we also expand $\psi(s), \theta(s)$, and $\phi(s)$ in powers of $\epsilon$. The boundary conditions for these Euler Angles are

$$
\begin{aligned}
\psi(0)=\pi / 2, \quad \theta(0) & =\pi / 2-\xi, \quad \phi(0)=\pi \\
\psi(1 / 2)=2 \pi+\pi / 2, \quad \theta(1 / 2) & =\pi / 2, \quad \phi(1 / 2)=\pi-\Delta \varphi / 2 .
\end{aligned}
$$

We solve (2.1), (2.2), (4.1), and (4.2) at each order. The solution at order 0 is given by (6.6) and

$$
\begin{aligned}
\mathbf{n}^{[0]} & =\mathbf{0}, \mathbf{m}^{[0]}=\mathbf{u}^{[0]}=4 \pi \mathbf{e}_{\mathbf{x}}, \mathbf{d}_{\mathbf{1}}{ }^{[0]}=\mathbf{e}_{\mathbf{x}}, \mathbf{d}_{\mathbf{2}}{ }^{[0]}=\mathbf{e}_{\theta}, \mathbf{d}_{\mathbf{3}}{ }^{[0]}=\mathbf{e}_{\tau} \\
\psi^{[0]}(s) & =\pi / 2+4 \pi s, \theta^{[0]}(s)=\pi / 2, \phi^{[0]}(s)=\pi, \xi^{[0]}=0, \delta^{[0]}=0
\end{aligned}
$$

This solution fulfills boundary conditions $(6.2)$ and we see that we have to either take $s_{c}^{[0]}=1 / 4$ or $s_{c}^{[0]}=1 / 2$ in order to fulfill the contact conditions $(6.3)$, with $s_{c}^{[0]}=1 / 4$ corresponding to curve I and $s_{c}^{[0]}=1 / 2$ corresponding to curve II, see Figure 12 .

The solution functions at order 1 are listed in the Appendix B. For curve I, the unknown parameters are

$$
\begin{aligned}
& n_{x}^{[1]}(0)=-\frac{8 \pi \beta}{\cos (\pi / \sqrt{2})}, \xi^{[1]}=\beta \frac{\tan (\pi / \sqrt{2})}{\sqrt{2}}, m_{\tau}^{[1]}(0)=2 \beta\left[\frac{\sqrt{2} \pi}{\tan (\sqrt{2} \pi)}-\frac{2}{\cos (\pi / \sqrt{2})}\right] \\
& m_{x}^{[1]}(0)=-8 \pi, n_{\tau}^{[1]}(0)=32 \pi^{2}, s_{c}^{[1]}=1 / 4, \delta^{[1]}=\frac{\beta}{2 \pi}\left(1-\frac{1}{\cos (\pi / \sqrt{2})}\right)=0.422 \beta
\end{aligned}
$$

Calculations at order 2 yields

$$
\delta^{[2]}=-\frac{\beta}{4 \pi}\left(\sqrt{2} \pi\left[\frac{2}{\tan (\sqrt{2} \pi)}+\frac{1}{\sin (\pi / \sqrt{2})}\right]-4\left[1+\frac{1}{\cos (\pi / \sqrt{2})}\right]^{2}\right)
$$

which finally yields

$$
\delta_{\mathrm{I}} \simeq \epsilon \delta^{[1]}+\epsilon^{2} \delta^{[2]}=0.422 \Delta \varphi-0.505 z^{*} \Delta \varphi
$$

For curve II, the unknown parameters are

$$
\begin{aligned}
& n_{x}^{[1]}(0)=-8 \pi \beta, \xi^{[1]}=\beta \frac{\tan (\pi / \sqrt{2})}{\sqrt{2}}, m_{\tau}^{[1]}(0)=2 \beta\left[-2+\frac{\sqrt{2} \pi}{\tan (\sqrt{2} \pi)}\right] \\
& m_{x}^{[1]}(0)=-8 \pi, n_{\tau}^{[1]}(0)=32 \pi^{2}, s_{c}^{[1]}=-1 / 2, \delta^{[1]}=0
\end{aligned}
$$

Calculations at order 2 yields $\delta^{[2]}=0$ and it is only at order 3 that a non-vanishing $\delta^{[3]}=(\pi / 2) \beta$ is found, yielding

$$
\delta_{\mathrm{II}} \simeq \epsilon^{3} \delta^{[3]}=(\pi / 2) z^{* 2} \Delta \varphi
$$

Comparing (6.15) and (6.18), we see that the intuitive picture of Figure 12 is confirmed: for given $z^{*}$, both branches are linear and meet at the origin, and the increased order $z^{* 2}$ in Branch II implies 

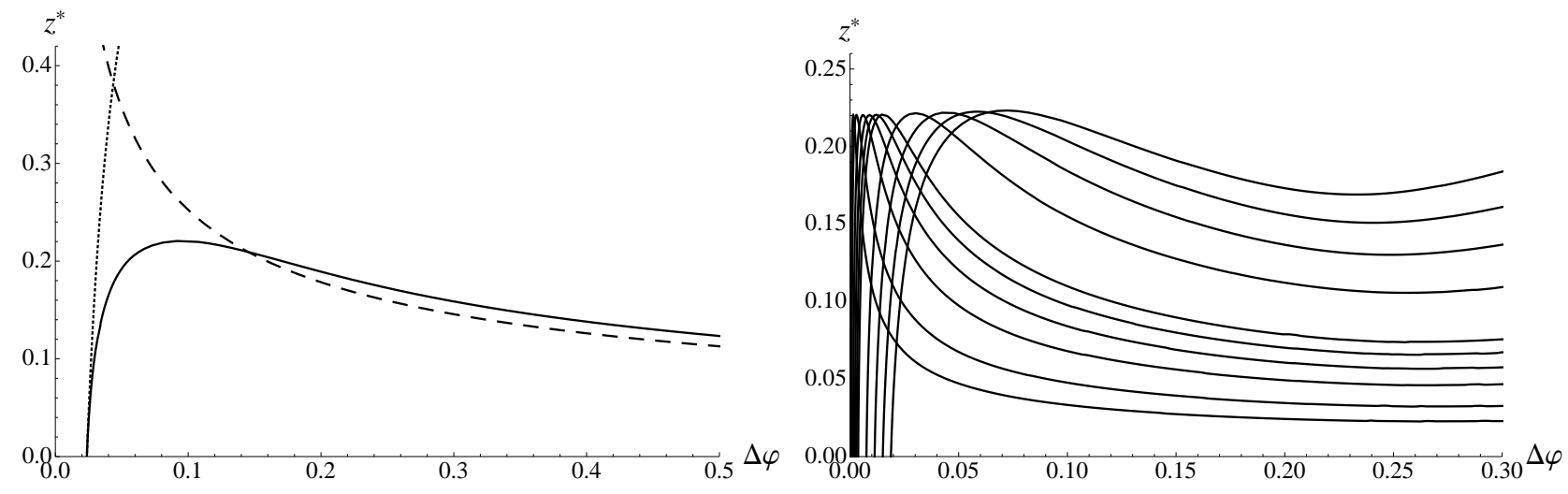

Figure 13: Left: For $\delta=0.01$, numerical bifurcation curve (solid) for configurations with one selfcontact point, in the Elastic Strip model, and its comparison with analytical approximations (6.15) (dotted curve) and (6.18) (dashed curve). Right: Numerical bifurcation curves for configurations with one self-contact point for the Elastic Strip model, for $\delta=0.05,0.04,0.03,0.020 .01,0.008$, $0.006,0.004,0.002,0.001$. The maximum $z^{*}$ value for the curves tends to $z^{*} \simeq 0.223$ as $\delta \rightarrow 0$.

a much shallower slope, with the wedge region between consisting of good knots. Moreover, the positive slope $(\pi / 2) z^{* 2}$ of $\delta_{\text {II }}$ means that Branch II always sits above the $\delta=0$ axis, providing further evidence that some degree of end-shift is necessary for a good knot.

An alternative way of viewing the analytical expressions is to fix $\delta$ and plot the boundary curves in the $\Delta \varphi-z^{*}$ plane. These analytical curves are compared with numerical computations in Figure 13-Left. The numerical computations follow configurations with one self-contact point, as for curve $X_{1}$ in Figure 7-right. The crossing of curve I and II happens at $z^{*}=0.382$, a value independent of $\delta$. This is an approximation of the maximum $z^{*}$ point on the numerical curve, see Figure 13-Left. We see in Figure 13-Right that on numerical curves, this maximum point only very slowly depends on $\delta$, and that as $\delta \rightarrow 0$, this point converge towards $z^{*} \simeq 0.223$. This limit value may be computed as the end-to-end distance of a self-contacting planar elastica, see [28] and Figure 14. It is the solution of

$$
\begin{aligned}
0 & =\mathcal{E}(3 \pi / 4, m)-\left(1-\frac{m}{2}\right) \mathcal{F}(3 \pi / 4, m) \\
z^{*} & =1-\frac{2}{m}\left[1-\frac{\mathcal{E}(\pi / 2, m)}{\mathcal{F}(\pi / 2, m)}\right]
\end{aligned}
$$

where $m \simeq-5.4, \mathcal{F}(x, m)$ is the incomplete elliptic integral of the first kind, and $\mathcal{E}(x, m)$ is the incomplete elliptic integral of the second kind.

\section{Discussion}

In this paper we have explored the possibility of generating stable knot configurations of an elastic rod or strip in a clamped geometry and with no points of self-contact. One motivation for this study was the simple observation that such configurations can be replicated with a strip of paper or 


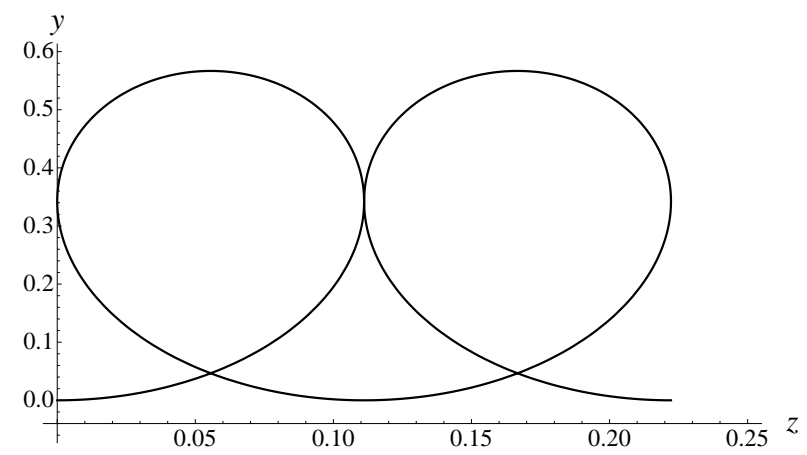

Figure 14: Self-contacting planar elastica corresponding to the limit case $\delta \rightarrow 0, \Delta \varphi \rightarrow 0$, and $z^{*}$ given by Eq. (6.20).

flexible wire, but that it can be quite difficult or even impossible, depending on the exact material used. Our theoretical analysis has confirmed the existence of such configurations, and we have quantitatively validated this experimentally in the case of a strip. We have generally considered the question in terms of a three-dimensional parameter space consisting of the natural degrees of freedom available when attempting to generate such a knot by hand: end-displacement, -shift, and -rotation; and have located regions of this parameter space for which the desired knots are found. Our analysis seems to confirm the fact that finding a good knot is easier with a strip than with, say, a flexible wire with circular cross-section. Indeed, we find that within Kirchhoff theory, the region of good knots generally is smaller than within a strip theory, and that the requirement on material properties for a stable knot within Kirchhoff theory are quite limiting.

Aside from the specific issue of contact-free knots, our analysis required the use of several computational and analytical techniques that can easily be adapted to other settings. The 18D system associated with an inextensible unshearable Kirchhoff rod presents a rich solution structure, with many equilibrium solutions for any given set of boundary conditions; hence locating a desired configuration - in particular one that is stable - can be a daunting task. The linear stability calculation can also be numerically challenging, requiring knowledge of the sign of all eigenvalues of a high-dimensional system. Adapting the constitutive equations to model a strip is straightforward in principle, but brings challenges of its own due to the nonlinearity introduced in the constitutive law (4.2). Here we have utilised a combination of numerical shooting, branch tracing, and asymptotic analysis; and have attempted to highlight any "tricks" employed to facilitate in computations.

There are several directions that could be further explored in this problem. In our macro-scale experiments, the effect of gravity was apparent, a feature that would be straightforward to add theoretically. If we relax the condition that the ends are aligned, this would add another parameter and must presumably generate a larger region of good knots. Also, the analysis presented here really only considers the "simplest" knot, the trefoil (including the closing loop at infinity). A similar analysis could in theory be performed with any higher knot genus, though the numerical computations may become increasingly difficult for more complex knots. On the other hand, we conjecture that in fact stable contact-free knot configurations exist in theory for any toroidal knot. This is a consequence of generalizing the arguments posed in Section 6 : for $K_{2}=K_{3}=K$, the 
$n$-covered ring is stable if $K \geq n$, and toroidal knots of increasing genus are (likely) nearby the $n$-covered ring. Of course, it remains to be seen via either formal asymptotic analysis about the $n$-covered ring for $n>2$ and/or numerical computation whether such regions of parameter space can explicitly be found. Here we restricted our analysis to the values $K_{2}=K_{3}=3$, a choice that ensured stability of the doubly-covered ring but that required a Poisson ratio of negative $1 / 2$ in an elliptical geometry. While fixing these parameters created a more manageable parameter space to explore, from a theoretical point of view toroidal good knots of a given genus will be attainable in a rod framework over regions of the $K_{2}-K_{3}$ space. Whether such regions are physically realizable is a separate issue: while $K_{2}$ can be computed for any geometry using simple formulae for second moment of area, and can in theory be made to take on any positive value, the torsional rigidity constant that determines $K_{3}$ is much more difficult to compute and has only been determined for a very small number of cross-section geometries [38, 23].

A final direction of interest for future study is to connect to polymer mechanics. As noted in the introduction, there is an abundance of knots found in polymers in biology, and in numerous instances the presence of self-contact is an important feature underlying the biological activity. Our analysis suggests that it may be possible for a knotted polymer to have no points of selfcontact, but that such situations would be rarely encountered; this raises the interesting questions of whether such configurations do occur and if so what is the impact on the biological function.

\section{Acknowledgements}

The authors thank A. Goriely for discussions and support, P. Pieranski for mentioning the problem of stable knots, and E. Starostin for providing the Lurie reference. S.N. acknowledges support from the U.K. Royal Society through the International Exchanges Scheme (grant IE120203), and from the french Agence Nationale de la Recherche through grants ANR-13-JS09-0009 and ANR14-CE07-0023-01.

\section{A Full system}

We provide here the full set of equations in component form for the dynamical system, equilibrium configurations, and the linear stability system. 
Dynamical system. The dynamical system consists of the force and moment balance (2.1), the moving frame equations, (2.2), as well as $\mathbf{r}^{\prime}=\mathbf{d}_{3}$. In component form these read

$$
\begin{array}{rll}
x^{\prime}=d_{3 x} & , & n_{1}^{\prime}=n_{2} u_{3}-n_{3} u_{2}-f_{1}+\rho A\left(\ddot{x} d_{1 x}+\ddot{y} d_{1 y}+\ddot{z} d_{1 z}\right) \\
y^{\prime}=d_{3 y} & , \quad & n_{2}^{\prime}=n_{3} u_{1}-n_{1} u_{3}-f_{2}+\rho A\left(\ddot{x} d_{2 x}+\ddot{y} d_{2 y}+\ddot{z} d_{2 z}\right) \\
z^{\prime}=d_{3 z} & , \quad & n_{3}^{\prime}=n_{1} u_{2}-n_{2} u_{1}-f_{3}+\rho A\left(\ddot{x} d_{3 x}+\ddot{y} d_{3 y}+\ddot{z} d_{3 z}\right) \\
d_{3 x}^{\prime}=u_{2} d_{1 x}-u_{1} d_{2 x} & , \quad & m_{1}^{\prime}=m_{2} u_{3}-m_{3} u_{2}+n_{2} \\
d_{3 y}^{\prime}=u_{2} d_{1 y}-u_{1} d_{2 y} & , & m_{2}^{\prime}=m_{3} u_{1}-m_{1} u_{3}-n_{1} \\
d_{3 z}^{\prime}=u_{2} d_{1 z}-u_{1} d_{2 z} & , \quad & m_{3}^{\prime}=m_{1} u_{2}-m_{2} u_{1} \\
d_{1 x}^{\prime}=u_{3} d_{2 x}-u_{2} d_{3 x} & , & d_{2 x}^{\prime}=u_{1} d_{3 x}-u_{3} d_{1 x} \\
d_{1 y}^{\prime}=u_{3} d_{2 y}-u_{2} d_{3 y} & , & d_{2 y}^{\prime}=u_{1} d_{3 y}-u_{3} d_{1 y} \\
d_{1 z}^{\prime}=u_{3} d_{2 z}-u_{2} d_{3 z} & , & d_{2 z}^{\prime}=u_{1} d_{3 z}-u_{3} d_{1 z} .
\end{array}
$$

Equilibrium system. Setting time derivatives to zero and denoting equilibrium variables with index $e$, we have the system

$$
\begin{aligned}
& x_{e}^{\prime}=d_{3 x e} \quad, \quad n_{1 e}^{\prime}=n_{2 e} u_{3 e}-n_{3 e} u_{2 e}-f_{1} \\
& y_{e}^{\prime}=d_{3 y e} \quad, \quad n_{2 e}^{\prime}=n_{3 e} u_{1 e}-n_{1 e} u_{3 e}-f_{2} \\
& z_{e}^{\prime}=d_{3 z e} \quad, \quad n_{3 e}^{\prime}=n_{1 e} u_{2 e}-n_{2 e} u_{1 e}-f_{3} \\
& d_{3 x e}^{\prime}=u_{2 e} d_{1 x e}-u_{1 e} d_{2 x e} \quad, \quad m_{1 e}^{\prime}=m_{2 e} u_{3 e}-m_{3 e} u_{2 e}+n_{2 e} \\
& d_{3 y e}^{\prime}=u_{2 e} d_{1 y e}-u_{1 e} d_{2 y e} \quad, \quad m_{2 e}^{\prime}=m_{3 e} u_{1 e}-m_{1 e} u_{3 e}-n_{1 e} \\
& d_{3 z e}^{\prime}=u_{2 e} d_{1 z e}-u_{1 e} d_{2 z e} \quad, \quad m_{3 e}^{\prime}=m_{1 e} u_{2 e}-m_{2 e} u_{1 e} \\
& d_{1 x e}^{\prime}=u_{3 e} d_{2 x e}-u_{2 e} d_{3 x e} \quad, \quad d_{2 x e}^{\prime}=u_{1 e} d_{3 x e}-u_{3 e} d_{1 x e} \\
& d_{1 y e}^{\prime}=u_{3 e} d_{2 y e}-u_{2 e} d_{3 y e} \quad, \quad d_{2 y e}^{\prime}=u_{1 e} d_{3 y e}-u_{3 e} d_{1 y e} \\
& d_{1 z e}^{\prime}=u_{3 e} d_{2 z e}-u_{2 e} d_{3 z e} \quad, \quad d_{2 z e}^{\prime}=u_{1 e} d_{3 z e}-u_{3 e} d_{1 z e}
\end{aligned}
$$

where

$$
u_{1 e}=m_{1 e}, \quad u_{2 e}=\frac{m_{2 e}}{K_{2}}, \quad u_{3 e}=\frac{m_{3 e}}{K_{3}} .
$$

Stability system. Following the perturbation scheme outlined in the main text, the eigenvalues $\omega$ that determine stability are determined from the following set of 12 equations

$$
\begin{array}{rll}
\bar{x}^{\prime}=\alpha_{2} d_{1 x e}-\alpha_{1} d_{2 x e} & , & \bar{n}_{1}^{\prime}=n_{2 e} \bar{u}_{3}+\bar{n}_{2} u_{3 e}-n_{3 e} \bar{u}_{2}-\bar{n}_{3} u_{2 e}-\omega^{2}\left(\bar{x} d_{1 x e}+\bar{y} d_{1 y e}+\bar{z} d_{1 z e}\right) \\
\bar{y}^{\prime}=\alpha_{2} d_{1 y e}-\alpha_{1} d_{2 y e} & , \quad \bar{n}_{2}^{\prime}=n_{3 e} \bar{u}_{1}+\bar{n}_{3} u_{1 e}-n_{1 e} \bar{u}_{3}-\bar{n}_{1} u_{3 e}-\omega^{2}\left(\bar{x} d_{2 x e}+\bar{y} d_{2 y e}+\bar{z} d_{2 z e}\right) \\
\bar{z}^{\prime}=\alpha_{2} d_{1 z e}-\alpha_{1} d_{2 z e} & , \quad & \bar{n}_{3}^{\prime}=n_{1 e} \bar{u}_{2}+\bar{n}_{1} u_{2 e}-n_{2 e} \bar{u}_{1}-\bar{n}_{2} u_{1 e}-\omega^{2}\left(\bar{x} d_{3 x e}+\bar{y} d_{3 y e}+\bar{z} d_{3 z e}\right) \\
\alpha_{1}^{\prime}=\bar{u}_{1}+u_{3 e} \alpha_{2}-u_{2 e} \alpha_{3} & , & \bar{m}_{1}^{\prime}=m_{2 e} \bar{u}_{3}+\bar{m}_{2} u_{3 e}-m_{3 e} \bar{u}_{2}-\bar{m}_{3} u_{2 e}+\bar{n}_{2} \\
\alpha_{2}^{\prime}=\bar{u}_{2}+u_{1 e} \alpha_{3}-u_{3 e} \alpha_{1} & , & \bar{m}_{2}^{\prime}=m_{3 e} \bar{u}_{1}+\bar{m}_{3} u_{1 e}-m_{1 e} \bar{u}_{3}-\bar{m}_{1} u_{3 e}-\bar{n}_{1} \\
\alpha_{3}^{\prime}=\bar{u}_{3}+u_{2 e} \alpha_{1}-u_{1 e} \alpha_{2} & , & \bar{m}_{3}^{\prime}=m_{1 e} \bar{u}_{2}+\bar{m}_{1} u_{2 e}-m_{2 e} \bar{u}_{1}-\bar{m}_{2} u_{1 e} .
\end{array}
$$




\section{B Asymptotic approach}

We here detail the asymptotic approach to compute the parameter value for which knotted configurations exist near the point $z^{*}=0, \Delta \varphi=0, \delta=0$, see Section 6. For Elastic Strips, the doubly covered ring is stable [3], while for Kirchhoff rods, stability of the $n$-covered ring has been established analytically [24] as a function of material parameters $K_{2}, K_{3}$, yielding the result that the configuration is stable if and only if

$$
\sqrt{\frac{K_{2}-1}{K_{2}} \frac{K_{3}-1}{K_{3}}} \geq \frac{n-1}{n} .
$$

In the case of the doubly-covered ring, with $n=2$, and with $K_{2}=K_{3}$, (B.1) simply reads $K_{2}=K_{3} \geq 2$ for stability. If this is satisfied, then since knotted configurations exist nearby, it is reasonable to expect that these knotted configurations will be stable. We introduce the notation $k_{2}=1-1 / K_{2}$ and $k_{3}=1-1 / K_{3}$ and we present results valid for both the Elastic Strip and Kirchhoff rod models, as well as for both curves I and II. (Formally, the Elastic Strip case is recovered by setting $k_{2}=1$ and $k_{3}=1 / 2$.) We uses $(X, Z, X)$ Euler angles $(\psi, \theta, \phi)$ where the material frame is given by

$$
\left\{\mathbf{d}_{\mathbf{1}}, \mathbf{d}_{\mathbf{2}}, \mathbf{d}_{\mathbf{3}}\right\}^{T}=\mathcal{M} \cdot\left\{\mathbf{e}_{\mathbf{1}}, \mathbf{e}_{\mathbf{2}}, \mathbf{e}_{\mathbf{3}}\right\}^{T}
$$

with

$$
\mathcal{M}=\left[\begin{array}{ccc}
-\cos \phi \sin \theta & \cos \theta \cos \phi \cos \psi-\sin \phi \sin \psi & \cos \psi \sin \phi+\cos \theta \cos \phi \sin \psi \\
\sin \theta \sin \phi & -\cos \theta \cos \psi \sin \phi-\cos \phi \sin \psi & \cos \phi \cos \psi-\cos \theta \sin \phi \sin \psi \\
\cos \theta & \cos \psi \sin \theta & \sin \theta \sin \psi
\end{array}\right]
$$

The solution at order 0 is given by (6.6), (6.10), and (6.11). At order 1, one finds the force to be $\mathbf{n}^{[1]}=\left(n_{x}^{[1]}(0), 0, n_{\tau}^{[1]}(0)\right)^{T}$ while the moment and Darboux vectors are

$$
\begin{array}{ll}
m_{x}^{[1]}(s)=m_{x}^{[1]}(0)+\frac{n_{\tau}^{[1]}(0)}{4 \pi}(1-\cos (4 \pi s)) & u_{x}^{[1]}(s)=m_{x}^{[1]}(s) \\
m_{t}^{[1]}(s)=\left(m_{\tau}^{[1]}(0)-\frac{n_{x}^{[1]}(0)}{4 \pi}\right) \sin (4 \pi s) & u_{t}^{[1]}(s)=4 \pi k_{2} \phi^{[1]}(s)+\left(1-k_{2}\right) m_{t}^{[1]}(s) \\
m_{\tau}^{[1]}(s)=\frac{n_{x}^{[1]}(0)}{4 \pi}+\left(m_{\tau}^{[1]}(0)-\frac{n_{x}^{[1]}(0)}{4 \pi}\right) \cos (4 \pi s) & u_{\tau}^{[1]}(s)=4 \pi k_{3} \theta^{[1]}(s)+\left(1-k_{3}\right) m_{\tau}^{[1]}(s)
\end{array}
$$


The Euler angles and position of the center line are

$$
\begin{aligned}
& \psi^{[1]}(s)=\left(\frac{n_{\tau}^{[1]}(0)}{4 \pi}+m_{x}^{[1]}(0)\right) s-\frac{n_{\tau}^{[1]}(0)}{16 \pi^{2}} \sin (4 \pi s) \\
& \theta^{[1]}(s)=\frac{m_{\tau}^{[1]}(s)}{4 \pi}-\frac{n_{x}^{[1]}(0)}{16 \pi^{2} k_{3}}+\left(\frac{n_{x}^{[1]}(0)}{4 \pi}-k_{3} m_{\tau}^{[1]}(0)-4 \pi k_{3} \xi^{[1]}\right) \frac{\cos \left(4 \sqrt{k_{2} k_{3}} \pi s\right)}{4 \pi k_{3}} \\
& \phi^{[1]}(s)=\frac{m_{t}^{[1]}(s)}{4 \pi}+\left(\frac{n_{x}^{[1]}(0)}{4 \pi}-k_{3} m_{\tau}^{[1]}(0)-4 \pi k_{3} \xi^{[1]}\right) \frac{\sin \left(4 \pi \sqrt{k_{2} k_{3}} s\right)}{4 \pi \sqrt{k_{2} k_{3}}} \\
& x^{[1]}(s)=\frac{1}{4 \pi k_{3}}\left(\frac{1-k_{3}}{4 \pi}\left[n_{x}^{[1]}(0) s+m_{t}^{[1]}(s)\right]-\phi^{[1]}(s)\right) \\
& y^{[1]}(s)=\left[m_{x}^{[1]}(s)+m_{x}^{[1]}(0)\right] \frac{\sin ^{2}(2 \pi s)}{16 \pi^{2}}-\psi^{[1]}(s) \frac{\sin (4 \pi s)}{4 \pi} \\
& z^{[1]}(s)=-\left[m_{x}^{[1]}(s)+m_{x}^{[1]}(0)+\frac{n_{\tau}^{[1]}(0)}{4 \pi}\right] \frac{\sin (4 \pi s)}{32 \pi^{2}}+\psi^{[1]}(s) \frac{\cos (4 \pi s)}{4 \pi}+\frac{n_{\tau}^{[1]}(0)}{32 \pi^{2}} s
\end{aligned}
$$

This solution fulfills the $s=0$ boundary conditions (given around equation (6.1) and in (6.8)). The parameters $n_{x}^{[1]}(0), n_{\tau}^{[1]}(0), m_{x}^{[1]}(0), m_{\tau}^{[1]}(0), \xi^{[1]}, s_{c}^{[1]}$, and $\delta^{[1]}$ are found by enforcing the $s=1 / 2$ boundary conditions (6.2) together with contact conditions (6.3):

$$
\begin{aligned}
n_{x}^{[1]}(0) & =2 \pi \beta \frac{\sin \left(4 \pi \sqrt{k_{2} k_{3}} s_{c}^{[0]}\right)}{\left(k_{3}-1\right) s_{c}^{[0]} \sin \left(2 \pi \sqrt{k_{2} k_{3}}\right)}, n_{\tau}^{[1]}(0)=32 \pi^{2}, m_{x}^{[1]}(0)=-8 \pi \\
m_{\tau}^{[1]}(0) & =\frac{n_{x}^{[1]}(0)}{4 \pi k_{3}}+\frac{2 \pi \beta \sqrt{k_{2} k_{3}}}{k_{3} \tan \left(2 \pi \sqrt{k_{2} k_{3}}\right)}, \xi^{[1]}=\beta k_{2} \frac{\tan \left(\pi \sqrt{k_{2} k_{3}}\right)}{2 \sqrt{k_{2} k_{3}}} \\
\delta^{[1]} & =\frac{\beta}{4 \pi k_{3}}-\frac{\beta \sin \left(4 \pi \sqrt{k_{2} k_{3}} s_{c}^{[0]}\right)}{8 \pi k_{3} s_{c}^{[0]} \sin \left(2 \pi \sqrt{k_{2} k_{3}}\right)}, s_{c}^{[1]}=-s_{c}^{[0]} / \cos \left(4 \pi s_{c}^{[0]}\right)
\end{aligned}
$$

with $s_{c}^{[0]}=1 / 4$ for curve I, and $s_{c}^{[0]}=1 / 2$ for curve II. For curve I, at order 2 , the shift is computed to be

$$
\delta^{[2]}=\frac{\beta \sin ^{-1}\left[(\pi / 2) \sqrt{k_{2} k_{3}}\right] \cos ^{-2}\left(\pi \sqrt{k_{2} k_{3}}\right)}{8 \pi k_{3}^{2}\left(-1+k_{3}\right)\left(-1+k_{2} k_{3}\right)\left(-1+4 k_{2} k_{3}\right)} \mathcal{P}\left(k_{2}, k_{3}\right)
$$

with $\mathcal{P}\left(k_{2}, k_{3}\right)$ given in Appendix C. For the classic case $k_{2}=k_{3}=2 / 3$ used in Section 3 , $\delta^{[2]}=-\beta[1 / \sqrt{3}+123 /(40 \pi)]$.

For curve II, we have

$$
\begin{aligned}
& \delta^{[2]}=0 \\
& \delta^{[3]}=k_{2}(\pi / 2) \beta
\end{aligned}
$$




\section{The function $\mathcal{P}\left(k_{2}, k_{3}\right)$}

The function $\mathcal{P}\left(k_{2}, k_{3}\right)$ introduced in $(\mathrm{B} .16)$ is

$$
\begin{aligned}
\mathcal{P}\left(k_{2}, k_{3}\right)= & p_{1}\left(k_{2}, k_{3}\right) \cos \left(\frac{1}{2} \pi \sqrt{k_{2} k_{3}}\right)+p_{2}\left(k_{2}, k_{3}\right) \cos \left(\frac{5}{2} \pi \sqrt{k_{2} k_{3}}\right)+ \\
& p_{3}\left(k_{2}, k_{3}\right) \sin \left(\frac{1}{2} \pi \sqrt{k_{2} k_{3}}\right)+p_{4}\left(k_{2}, k_{3}\right) \sin \left(\frac{3}{2} \pi \sqrt{k_{2} k_{3}}\right)+ \\
& p_{5}\left(k_{2}, k_{3}\right) \sin \left(\frac{5}{2} \pi \sqrt{k_{2} k_{3}}\right)
\end{aligned}
$$

with

$$
\begin{aligned}
& p_{1}\left(k_{2}, k_{3}\right)=-\pi \sqrt{k_{2} k_{3}}\left(-1+k_{3}\right) k_{3}\left(1-5 k_{2} k_{3}+4 k_{2}^{2} k_{3}^{2}\right) \\
& p_{2}\left(k_{2}, k_{3}\right)=p_{1}\left(k_{2}, k_{3}\right) \\
& p_{3}\left(k_{2}, k_{3}\right)=-10+\left(19+46 k_{2}\right) k_{3}-\left(8+99 k_{2}+36 k_{2}^{2}\right) k_{3}^{2}+16 k_{2}\left(3+5 k_{2}\right) k_{3}^{3}-40 k_{2}^{2} k_{3}^{4} \\
& p_{4}\left(k_{2}, k_{3}\right)=5-11\left(1+2 k_{2}\right) k_{3}+\left(7+62 k_{2}+20 k_{2}^{2}\right) k_{3}^{2}-15 k_{2}\left(3+4 k_{2}\right) k_{3}^{3}+44 k_{2}^{2} k_{3}^{4} \\
& p_{4}\left(k_{2}, k_{3}\right)=\left(-1+k_{3}\right)\left(1-\left(1+4 k_{2}\right) k_{3}+3 k_{2} k_{3}^{2}+4 k_{2}^{2} k_{3}^{3}\right)
\end{aligned}
$$

\section{A generic configuration}

We here give full details on the good-knot configuration shown in Figure 6-right. Initial values are $n_{1}(0)=-24.743, n_{2}(0)=17.772, n_{3}(0)=37.145, m_{1}(0)=7.5317, m_{2}(0)=-8.7100$, $m_{3}(0)=4.529$. Parameters are $K=3, z^{*}=0.115, \delta=0.05, \varphi /(2 \pi)=0.802, T w=0.107$, $\mathcal{W}_{p}^{\star}=3.695$.

\section{E A non-flip-symmetric configuration}

We here give full details on a configuration which is not flip-symmetric. The initial values are $n_{1}(0)=-52.49, n_{2}(0)=4.12, n_{3}(0)=7.84, m_{1}(0)=2.21, m_{2}(0)=-17.59, m_{3}(0)=10.01$. The solution has $K=3, z^{*}=0.15, \delta=0.05, \varphi /(2 \pi)=0.785, T w=0.23, \mathcal{W}_{p}^{\star}=3.554$, and is shown in Figure 15. The configuration is stable, knotted, and free of self-contact, but does not lie between walls.

\section{References}

[1] Javier Arsuaga, Mariel Vázquez, Sonia Trigueros, De Witt Sumners, and Joaquim Roca. Knotting probability of dna molecules confined in restricted volumes: Dna knotting in phage capsids. Proceedings of the National Academy of Sciences of the USA, 99(8):5373-5377, 2002.

[2] B. Audoly, N. Clauvelin, and S. Neukirch. Elastic knots. Physical Review Letters, 99(16):164301, 2007. 


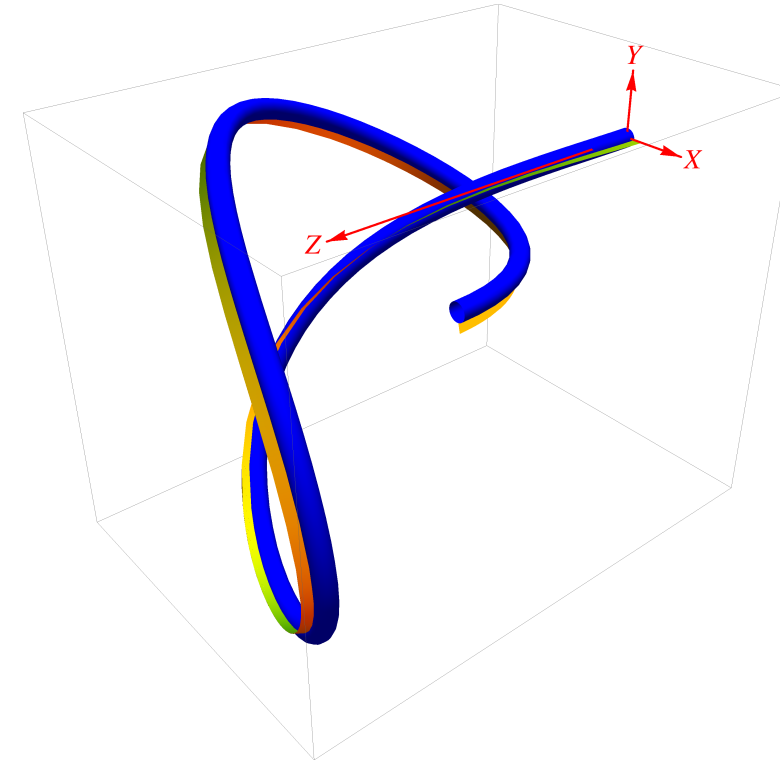

Figure 15: A non-flip-symmetric configuration which is stable, knotted, and free of self-contact, but which does not lie between walls.

[3] Basile Audoly and Keith A. Seffen. Buckling of naturally curved elastic strips: The ribbon model makes a difference. Journal of Elasticity, 119(1):293-320, 2015.

[4] Mitchell A Berger and Chris Prior. The writhe of open and closed curves. Journal of Physics A: Mathematical and General, 39(26):8321-8348, 2006.

[5] Miklós Bergou, Max Wardetzky, Stephen Robinson, Basile Audoly, and Eitan Grinspun. Discrete elastic rods. ACM Transactions on Graphics (SIGGRAPH), 27(3):63:1-63:12, 2008.

[6] N. Clauvelin, B. Audoly, and S. Neukirch. Matched asymptotic expansions for twisted elastic knots: A self-contact problem with non-trivial contact topology. Journal of the Mechanics and Physics of Solids, 57(9):1623-1656, 2009.

[7] B. D. Coleman and D. Swigon. Theory of supercoiled elastic rings with self-contact and its application to DNA plasmids. Journal of Elasticity, 60:173-221, 2000.

[8] David J. Craik, Norelle L. Daly, Trudy Bond, and Clement Waine. Plant cyclotides: a unique family of cyclic and knotted proteins that defines the cyclic cystine knot structural motif. Journal of molecular biology, 294(5):1327-1336, 1999.

[9] Marcelo A. Dias and Basile Audoly. A non-linear rod model for folded elastic strips. Journal of the Mechanics and Physics of Solids, 62:57-80, 2014.

[10] Marcelo A. Dias and Basile Audoly. 'Wunderlich, meet Kirchhoff': A general and unified description of elastic ribbons and thin rods. Journal of Elasticity, 119(1-2):49-66, 2015. 
[11] D.J. Dichmann, Y. Li, and J.H. Maddocks. Hamiltonian formulations and symmetries in rod mechanics. In J.P. Mesirov, K. Schulten, and D.W. Sumners, editors, Mathematical Approaches to Biomolecular Structure and Dynamics, volume 82 of The IMA Volumes in Mathematics and Its Applications, pages 71-113. Springer Verlag, 1996.

[12] G. Domokos and T. Healey. Hidden symmetry of global solutions in twisted elastic rings. Journal of Nonlinear Science, 11:47-67, 2001.

[13] Roger Fosdick and Eliot Fried, editors. The Mechanics of Ribbons and Moebius Bands. Springer, 2015.

[14] Alain Goriely, Michel Nizette, and Michael Tabor. On the dynamics of elastic strips. Journal of Nonlinear Science, 11(1):3-45, 2001.

[15] Alain Goriely and Michael Tabor. New amplitude equations for thin elastic rods. Phys. Rev. Lett., 77(17):3537-3540, 1996.

[16] Sachin Goyal, N. C. Perkins, and Christopher L. Lee. Non-linear dynamic intertwining of rods with self-contact. International Journal of Non-Linear Mechanics, 43(1):65-73, 2008.

[17] T. J. Healey. Material Symmetry and Chirality in Nonlinearly Elastic Rods. Mathematics and Mechanics of Solids, 7(4):405-420, 2002.

[18] Sophie E Jackson, Antonio Suma, and Cristian Micheletti. How to fold intricately: using theory and experiments to unravel the properties of knotted proteins. Current Opinion in Structural Biology, 42:6-14, 2017.

[19] Michal Jamroz, Wanda Niemyska, Eric J. Rawdon, Andrzej Stasiak, Kenneth C. Millett, Piotr Sukowski, and Joanna I. Sulkowska. Knotprot: a database of proteins with knots and slipknots. Nucleic Acids Research, 43(D1):D306-D314, 2015.

[20] M. K. Jawed, P. Dieleman, B. Audoly, and P. M. Reis. Untangling the mechanics and topology in the frictional response of long overhand elastic knots. Phys. Rev. Lett., 115(11):118302, 2015.

[21] Joel Langer and David A Singer. Knotted Elastic Curves in IR3. J. London Math. Soc.(2), 30:512-520, 1984.

[22] Nicole C H Lim and Sophie E Jackson. Molecular knots in biology and chemistry. Journal of Physics: Condensed Matter, 27(35):354101, 2015.

[23] A. I. Lurie. Theory of elasticity. Foundations of Engineering Mechanics. Springer, 2005.

[24] Robert S. Manning and Kathleen A. Hoffman. Stability of n-covered circles for elastic rods with constant planar intrinsic curvature. Journal of elasticity and the physical science of solids, 62(1):1-23, 2001. 
[25] Davide Marenduzzo, Cristian Micheletti, Enzo Orlandini, and De Witt Sumners. Topological friction strongly affects viral dna ejection. Proceedings of the National Academy of Sciences of the USA, 110(50):20081-20086, 2013.

[26] Sébastien Neukirch, Joël Frelat, Alain Goriely, and Corrado Maurini. Vibrations of postbuckled rods: The singular inextensible limit. Journal of Sound and Vibration, 331(3):704 $720,2012$.

[27] Sébastien Neukirch and Michael E. Henderson. Classification of the spatial clamped elastica: symmetries and zoology of solutions. Journal of Elasticity, 68:95-121, 2002.

[28] M. Nizette and A. Goriely. Toward a classification of Euler-Kirchhoff filaments. Journal of Mathematical Physics, 40:2830-2866, 1999.

[29] P. Patricio, M. Adda-Bedia, and M. Ben Amar. An elastica problem: instabilities of an elastic arch. Physica D, 124:285-295, 1998.

[30] P. Pieranski, S. Przybyl, and A. Stasiak. Tight open knots. The European Physical Journal E, 6:123-128, 2001.

[31] Christopher B. Prior and Sébastien Neukirch. The extended polar writhe: a tool for open curves mechancis. J. Phys. A: Math. Theo., 49:215201, 2016.

[32] V. Rossetto and A. C. Maggs. Writhing geometry of open DNA. Journal of Chemical Physics, 118(21):9864-9874, 2003.

[33] F. Schuricht and H. von der Mosel. Euler-Lagrange equations for nonlinearly elastic rods with self-contact. Archive for Rational Mechanics and Analysis, 168(1):35-82, 2003.

[34] E. L. Starostin. Equilibrium configurations of a thin elastic rod with self-contacts. PAMM, Proc. Appl. Math. Mech., 1(1):137-138, 2002.

[35] E. L. Starostin and G. H. M. van der Heijden. The shape of a mobius strip. Nature Materials, 6(8):563-567, 2007.

[36] Joanna I. Sulkowska, Piotr Sulkowski, P. Szymczak, and Marek Cieplak. Stabilizing effect of knots on proteins. Proceedings of the National Academy of Sciences, USA, 105(50):1971419719, 2008.

[37] D. W. Sumners and S. G. Whittington. Knots in self-avoiding walks. J. Phys. A: Math. Gen., 21:1689-1694, 1988.

[38] S. Timoshenko and J.N. Goodier. Theory of elasticity. New York, 1951.

[39] G. H. M van der Heijden, S. Neukirch, V. G. A. Goss, and J. M. T. Thompson. Instability and self-contact phenomena in the writhing of clamped rods. Int. J. Mech. Sci., 45(1):161-196, 2003. 
[40] Jeremiah R. Wagner, Joseph S. Brunzelle, Katrina T. Forest, and Richard D. Vierstra. A lightsensing knot revealed by the structure of the chromophore-binding domain of phytochrome. Nature, 438:325-331, 2005.

[41] Dungan J. M. Wasserman S. A. and Cozzarelli N. R. Discovery of a predicted DNA knot substantiates a model for site-specific recombination. Science, 22:171-174, 1985. 